
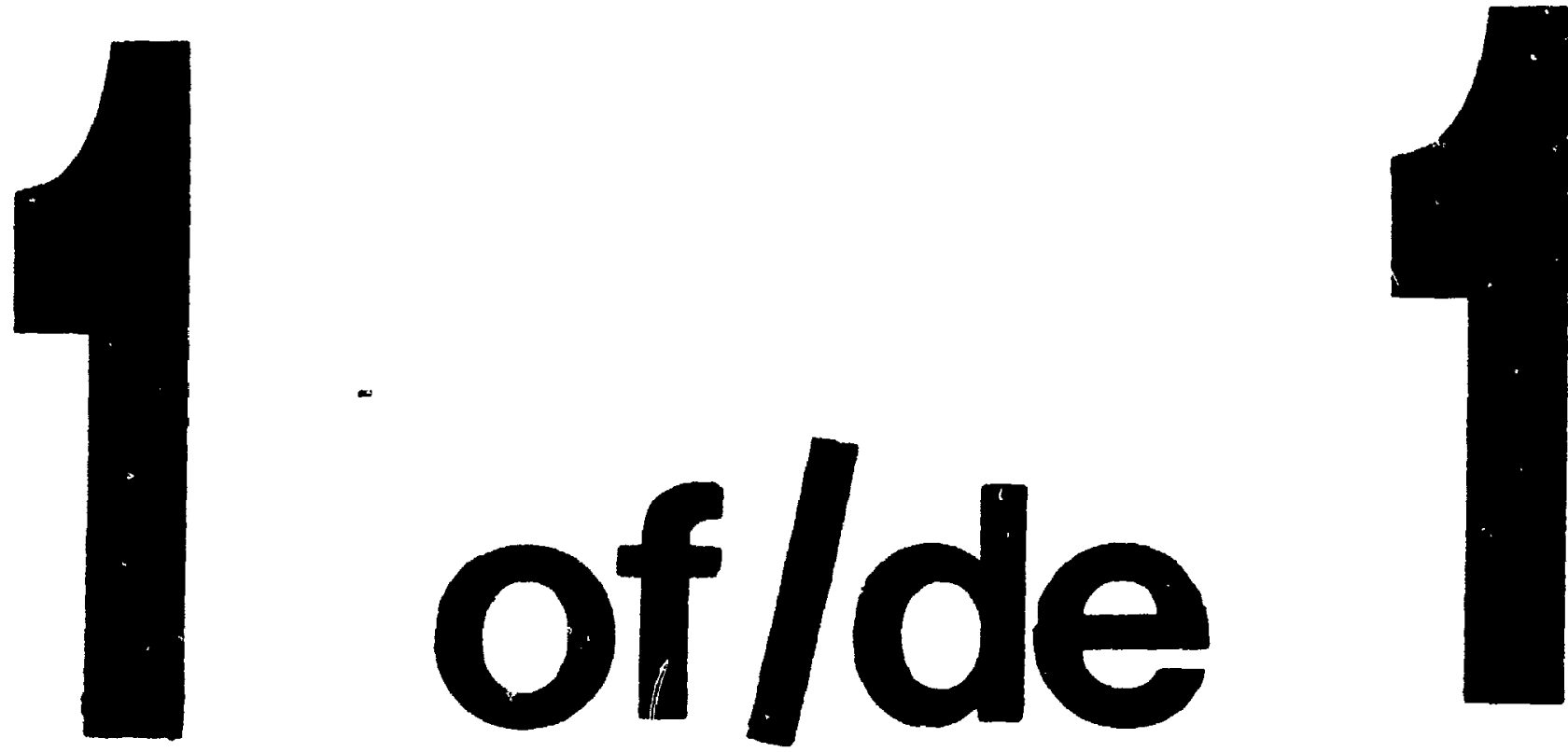

PM-1 33:2"X4" PHOTOGRAPHIC MICROCOPY TARGET NBS 1010a ANSI/ISO \#2 EQUIVALENT

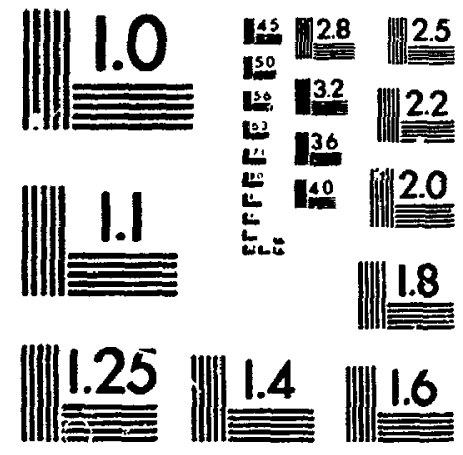

PAECISIONSM RESOLUTION TARGETS 
National Library

of Canada

Acquisitions and

Bibliographic Services Branch

395 Wellington Street

Ottawa Ontario

KIA ONA
Bibliotheque nationale

du Canada

Direction des acquisitions et

des senvices bibliographiques

395, rue Wellington

Oltawa (Ontario)

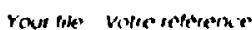

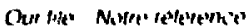

NOTICE

AVIS

The quality of this microform is heavily dependent upon the quality of the original thesis submitted for microfilming. Every effort has been made to ensure the highest quality of reproduction possible.

If pages are missing, contact the university which granted the degree.

Some pages may have indistinct print especially if the original pages were typed with a poor typewriter ribbon or if the university sent us an inferior photocopy.

Reproduction in full or in part of this microform is governed by the Canadian Copyright Act, R.S.C. 1970, c. C-30, and subsequent amendments.
La qualité de cette microforme dépend grandement de la qualité de la thèse soumise au microfilmage. Nous avons tout fait pour assurer une qualité supétieure de reproduction.

S'il manque des pages, veuillez communiquer avec l'université qui a conféré le grade.

La qualité d'impression de certaines pages peut laisser à désirer, surtout si les pages originales ont été dactylographiées a l'aide d'un ruban usé ou si l'université nous a fait parvenir une photocopie de qualité inférieure.

La reproduction, même partielle, de cette microforme est soumise à la Loi canadienne sur le droit d'auteur, SRC 1970, c. C-30, et ses amendements subséquents. 


\section{A Study of the Correlation States of Ethylene by Synchrotron Photoelectron Spectroscopy}

by

Steven J. Desjardins, B.Sc.

A thesis submitted to

the Faculty of Graduate Studies and Research

in partial fulfillment of

the requirements for the degree of

Master of Science

Department of Chemistry

Carleton University

Ottawa, Ontario

July 18, 1994

(C) copyright

1994, Steven J. Desjardins 
National Library

of Canada

Acquisitions and

Bibliographic Services Branch

395 Wellington Street Ottawa. Ontario KIAONA
Bibliotheque nationale du Canada

Direction des acquisitions et des services bibliographiques

395. rue Wellington

Ottawa (Ontario)

KIAON4
You hio Volve restence

Our the Norre coterence
THE AUTHOR HAS GRANTED AN IRREVOCABLE NON-EXCLUSIVE LICENCE ALLOWING THE NATIONAL LIBRARY OF CANADA TO REPRODUCE, LOAN, DISTRIBUTE OR SELL COPIES OF HIS/HER THESIS BY ANY MEANS AND IN ANY FORM OR FORMAT, MAKING THIS THESIS AVAILABLE TO INTERESTED PERSONS.
L'AUTEUR A ACCORDE UNE LICENCE IRREVOCABLE ET NON EXCLUSIVE PERMETTANT A LA BIBLIOTHEQUE NATIONALE DU CANADA DE REPRODUIRE, PRETER, DISTRIBUER OU VENDRE DES COPIES DE SA THESE DE QUELQUE MANIERE ET SOUS QUELQUE FORME QUE CE SOIT POUR METTRE DES EXEMPLAIRES DE CETTE THESE A LA DISPOSITION DES PERSONNE INTERESSEES.
THE AUTHOR RETAINS OWNERSHIP OF THE COPYRIGHT IN HIS/HER THESIS. NEITHER THE THESIS NOR SUBSTANTIAL EXTRACTS FROM IT MAY BE PRINTED OR OTHERWISE REPRODUCED WITHOUT HIS/HER PERMISSION.
LAATEUR CONSERVE LA PROPRIETE DU DROIT D'AUTEUR QUI PROTEGE SA THESE. NI LA THESE NI DES EXTRAITS SUBSTANTIELS DE CELLECI NE DOIVENT ETRE IMPRIMES OU AUTREMENT REPRODUITS SANS SON AUTORISATION. 
The undersigned recommend to the Faculty of Ciraduate Studies and Research acceptance of the thesis

\section{A Study of the Correlation States of Ethylene by Svnchrotron Photoelectron Spectroscopy}

submitted by Steven J. Desjardins, B.Sc. in partial fulfillment of the requirements for

the degree of Master of Science

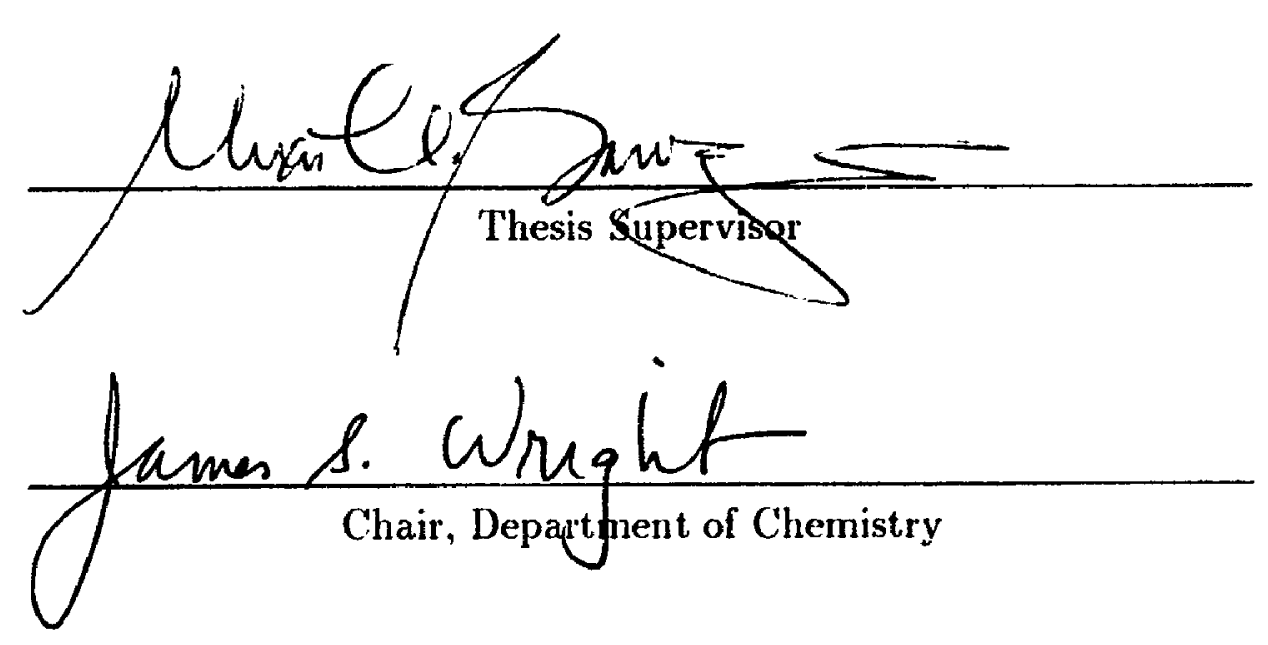

Carleton University

August 17, 1994 


\begin{abstract}
The synchrotron plotoelect ron spectra (SPES) of ethylene and $1{ }^{13} \mathrm{C}$ ethylene have been obtained over the photon energy range 30 to $220 \mathrm{fV}$. Two correlation (satellite) peaks are found in the spectra of ethylene and ${ }^{1-13} \mathrm{C}$-ethylene. 'The major satellite at $2 \pi \cdot 4 \mathrm{eV}$ binding energy is found to be intrinsic in nature, having no photon energy dependence in its intensity ratio with respect to its parent peak. The newly discovered peak at $21.4 \mathrm{eV}$ binding energy, however. is found to be dynamic. its intensity having a definite dependence on photon energy.
\end{abstract}




\section{Acknowledgements}

I would like to thank the Faculty. Staff and Students of the ('hernistry Department for all their help over the years. My family also deserves thanks for their encouragement and support. And, of course, my wife Seleena destrrees so much more than simple words could say, but I will simply have to sal I Lowe You and Thank You for Everything. 


\section{Terhnical Note}

This thesis document was typeset by the author using $\mathrm{LT}_{\mathrm{E}} \mathrm{X}$ and $\mathrm{PCT} \mathrm{E}_{\mathrm{X}}$

(Personal TEX Inc.). (iraphs were prodlsced using Graftool (3D visions Corp.). 


\section{Contents}

1 Introduction 1

1.1 Synchrotron Photoelectron Sipectroscopy ............. 1

1.2 Correlation States . . . . . . . . . . . . . . .

1.3 Ethylene ...................... s

2 Experimental $\quad 14$

3 Analysis $\quad 23$

4 Results and Discussion 25

4.1 The Intrinsic Correlation State . . . . . . . . . . . . 25

4.2 The Dynamic Correlation State ................ 34

5 Theoretical Calculations $\quad 5$

6 Conclusions $\quad 66$

7 Appendix A: Second Order Radiation 68

8 Appendix B: Ethylene Difference Spectrum $\quad 71$

$\begin{array}{lll}9 & \text { References } & \mathbf{7 3}\end{array}$ 


\section{List of Figures}

1 The Fthylene Molecule . . . . . . . . . . . . . 10

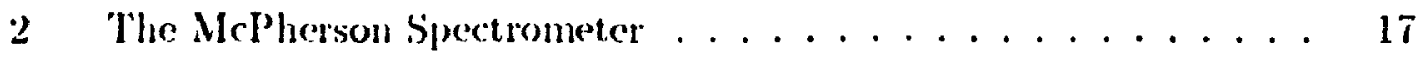

3 The Nladdlin Storage Ring . . . . . . . . . . . . 18

1 Ethylene Spectrum $(91.1,1) \ldots \ldots \ldots \ldots$

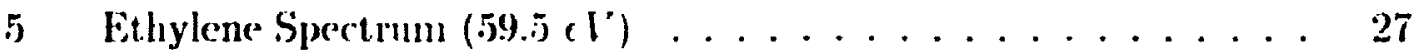

$6{ }^{13} \mathrm{C}$-Ethylene Spectrum $(59.5 \mathrm{eV}) \ldots \ldots \ldots \ldots \ldots$

7 Intensity Ratio of major satellite to $2 a_{g}^{-1}$ main peak . . . . . 31

8 Intensity Ratio of major satellite for ${ }^{13} \mathrm{C}$-Ethylene . . . . . . 33

9 Ethylene Spectrum $(49.5 \in V) \ldots \ldots \ldots \ldots \ldots \ldots$

$10{ }^{13} \mathrm{C}$-Ethylene Spectrum $(49.5 \mathrm{eV}) \ldots \ldots \ldots \ldots \ldots \ldots$

11 Intensity Ratio of the new correlation peak to $2 b_{1 u}^{-1}$ main peak . . 39

12 Intensity Ratio of the new correlation peak for ${ }^{13} \mathrm{C}$-Ethylene . . 41

13 Decomposition of Figure $11 \ldots \ldots \ldots \ldots$

14 The Oscillating Component of Figure $11 \ldots \ldots \ldots \ldots$

15 FFT of Oscillating Component $\ldots \ldots \ldots \ldots \ldots \ldots$

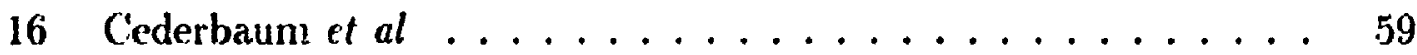

17 Murray and Davidson . . . . . . . . . . . . . 61

18 Wang and Davidson $\ldots \ldots \ldots \ldots \ldots \ldots \ldots$

19 Second Order Radiation $\ldots \ldots \ldots \ldots \ldots$

20 Difference Spectrum . . . . . . . . . . . . . 72 


\section{List of Tables}

1 Intensity Ratios for the major corrclation peak . . . . . . . . 30

2 Intensity Ratios for ${ }^{13}$ ('-Ethylene (najor) . . . . . . . . . . .32

3 Intensity Ratios for the new correlation prak . . . . . . . . 3N

4 Intensity Ratios for ${ }^{13}$ ('-Ethylene (new) . . . . . . . . . . . II

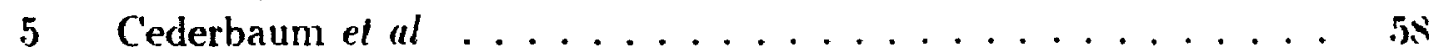

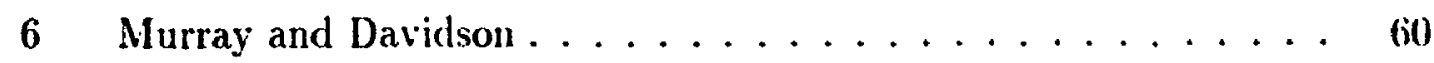

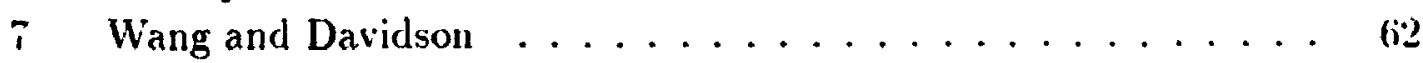

8 Second Order Radiation ................. (i9) 


\section{Introduction}

\subsection{Synchrotron Photoelectron Spectroscopy}

Photenelectron spectroscopy is the simplest and most direct method for the determination of the binding chergies of electrons in atomic and molecular species. Photons of a known energy, $h \nu$, interact with the sample $(M)$ under investigation. causing single ionization $\left(\mathrm{M}^{+}\right)$to a large extent. The ejected electrons, called photoelectrons, can be detected via their kinetic energies. $E_{K}$. The binding energies. $E_{B}$, can then easily be calculated by noting that:

$$
E_{h i}=h \nu-E_{B i}
$$

where the index $i$ is used to indicate that there nay be more than one $E_{K}$ and $E_{B}$ energy pair, thus reflecting the possibility of ionization out of more than one energy level of the species being studied. A typical experiment involves selecting an appropriate photon energy to cause the ionizations, and then counting the number of photoelectrons at a specific kinetic energy. A range of kinetic energies is then scanned to produce a complete spectrum. The kinetic energy spectrum can then be converted into a binding energy spectrum through the simple equation above. Binding energies can also be estimated using other techniques, like mass spectrometry, Penning ionization, electron impact spectroscopy, etc., but these experiments are rather complicated and the results are obtained indirectly, and thus are not as conclusive as photoelectron spectroscopy. 
The device that allows the photoedectron spectrum to be obtained is called a photoelectron spect rometer. I npectrometer consists of a collision region where the photons from the sonce interact with the sample being studied, an energy analysis section and a detection region where the photoclectrons are deterted or counted. The photoelectrons generally travel from the collision region to the detector through a guiding electric field which serves as the energy analyzer. The geometry of the energy analyzer region and the strength of the clectric and/or magnetic field will select the appropriate kinetic energy that will pass to the detector. The strength of the electric and/or magnetic field is adjustable, allowing for a range of kinetic energies to be detected and scamned over. Detectors generally work on a "cascade" type of process - the impinging photoelectrons cause a current to be generated and this current is then registered electronically.

Chargec particles radiate when accelerated. Acceleration can be a change in speed and/or a change in direction. If electrons are accelerated to relativistic speeds (ie $v / c \approx 1$ ) and then confined to an approxinately circular orbit through the use of bending magnets, they will radiate. As the electrons pass through the bending magnets, they radiate a photon beam tangentially to their path of travel that is very intense and well-focussed. This beam is called symchrotron radiation and the circular orbit is referred to as the syrchirotron ring [1]. Typical synchrot ron beams contain photons over a wide energy range, orders of magnitude in encrgy ( $<1$ to 1000 s of $e V$ ). Because this beam is intense (many photons), well-focussed 


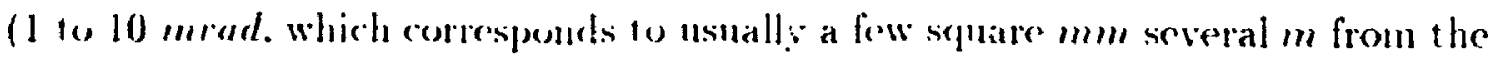
ynchrotron ring) and contains a wide (nergy range. sychchrot ron radiation is extremely useful for a wide wariety of experimental techniques, such as spectroscopy. diffraction and microscopy [2,3]. The ('anadian Synchrotron Radiation Facility (CSKF) has been used to obtain high resolution contact micrograph replicas of biological samples which yielded different information than that obtainable from conventional light microscops [2]. Reference 3 presents a review of the X-ray research being conducted at CSRF. Included are EXAFS, NEXAFS, Auger studies, surface studies, lithography and spectroscopy. And, many high-technology companies, including IBM, have used synchrotron radiation in the manufacture of small-scale $(\mu m)$ circuitry [3].

Synchrotron radiation is extremely valuable as a photon source for photoelectron spectroscopy for exactly the same three reasons. The intensity of the beam leads to many ionizations and hence more photoelectrons will bs produced. The small beam size allows for good alignment with the experimintal chamber and redures the loss of photons from scattering. And the wide energy range allows for the spectra of atomic or molecular species to be studied at different photon energies, a distinct advantage over discrete sources, such as conventional X-rays. Synchrotron radiation has other unique properties [1]. In the plane of its orbit, the radiation emitted by an electron is completely polarized with the electric vector parallel to the orbital plane [1]. Thus, experiments can be conducted at known 
angles to the polarization of the somer, like the magic angle [.t]. Filect rons travel around the synchrot ron ring in groups or bunches, and so, the radiation is emitted in short flashes or pulses [1]. However. the intensity from bunch to bunch is very stable, allowing for consistency in experimentation [1]. The time structure of the synchrotron radiation also allows for various types of time-resol ied experimental studies [1].

\subsection{Correlation States}

Simple single-particle molecular orbital models, like Hartree-Fock methors v. hich estimate the wavefunctions of many-electron systems by multiplying one-electron wavefunctions together, consider electron motions to be independent of each other. These models are successful at predicting the main features of valence shell and inner shell photoelectron spectra, though they cannot account for the "extra" peaks that are often observed. These extra peaks that appear in photoelectron spectra were initially called many-body states, satellite peaks or shake-up praks, though the more proper name correlation peaks is preferable as it indicates the mechanism responsible for these features. If simple molerular orbital theory as applied to the ground state of a neutral molecule is extended by considering excited state configurations, the result. called Configuration Interaction (C;), allows for the "mixing" or interacting of states in addition to the ground state configuration. It is precisely from the interactions (correlations) of electrons in atomic or molecular systems that these correlation peaks appear in the photoelectron spec- 
tra of many species $[\overline{2}-31]$. The comelation states of atoms, especially the noble gas atoms. have been investigated intensely, however the study of these states in molecular systems is still in its infancy. The work presented in this thesis is perhaps one of the most thorough investigations of the correlation peaks of a simple, non-dialomic molecuie.

The Schrödinger equation canmot be solved exactly for more than one electron in a system, and so, schemes were developed to estimate solutions of more complicated systems. As noted above, however, these schemes still considered electrons to be independent of each other. With the advances in experimental instrumentation and technique came the study of correlation peaks in photoelectron spectra as they could finally be resolved. It became increasingly obvious that electron interactions in atomic and molecular systems were not insignificant and a lack of understanding of the small features does imply an incomplete understanding of the whole. Theories involving the interactions of electrons, both in the system and in the continuum, were devised. Earlier calculations were unfortunately limited by computing power. But, with the recent advances in central processing unit power and speed and parallel computing, it is now possible to predict theoretically (within well-defined kinematical assumptions) what a photoelectron spectrum should look like, including correlation peaks.

Correlation peaks have everal production mechanisms [5,32]. Initial and Final Ionic State Configuration Interactions (ISCI and FISCI) occur when states 
of the same ormanetry interact in either the initial or final state's and these interactions are independent of the photoelectron energy. ('ontinum State (ontiguration Interaction (CSC'I), or inclastic scattering. involves the scattering of the photoelectron in the ionic potential. causing the excitation of another electron as the photoelectron leaves the system. Interchannel conpling, related to ('SC'I, give's rise to the autoionization of doubly excited states into different satellite channels. Shake-up is the process in which photoionization auses a relaxation of the system during and after the photoionization process, resulting in an excitation. Shake-off is the process where the second elertron leaves the system as well. 'The extension of the shake-up concept to continuum states is called conjugate shake-up.

Schemes have been proposed, such as those by Becker and Shirley [5], to classify correlation states and their production mechanisms phenomenologically by studying the photon energy dependence of the ratios of the correlation (satellite) peak intensities to the main or "parent" peaks observed in the photockectron spectra of noble gas atoms. The "parent" peak is the "normal" peak (one rlectron process) in the photoelectron spectrum expected within a Koopmans-type approximation. The photon energy dependence of satellite/main peak intensities in photoelectron spectra has been studied previously by Wuilleumier and Kranse [6]. The earlier X-ray studies were hampered by poor energy resolution and nany closely-spaced satellites were not resolved. With the introduction of high intensity synchrotron sources, such photon energy dependence studies have now led 
to loss ambignous morsinements of the satellite/main peak intensities. Those correlation (satellite) peatis that exhibit a constant watio with increasing photon nenergy are refored to as "intrinsic" comelations and are considered to be caused by initial and/or final state configuration interaction $[5,90.93]$; whereas those correlation (satellite) peaks that exhibit a strong photon energy dependence in the satellite to main peak intensity ratio are referred to as "dynamic" correlations and can be produced by shake-up processes $[5, \vec{\imath}-10.33]$. continuum state interactions $[5,9.11,12]$ or interchannel coupling $[5,8,9,3 \cdot 1]$

The terms "intrinsic" and "dynamic" are used because of their relation to the dependence on photon energy: Intrinsic correlation processes are independent of the photon energy of the ionization. and of the ionization itself. These processes are theoretically occuring whether there is ionization or not. And so, these processes are "constant" or "natural" and are therefore referred to as intrinsic. Dynamic correlation processes do depend on the energy of the ionization process. The name dynamic derives from the fact that these types of processes depend on the "dynamism" or speed (kinetic energy) of the departing photoelectron. And hence, they then depend on the photon energy because of the simple relationship between $E_{K}$ and $E_{B}$.

Several experimental and theoretical studies on atomic systems [5-24] have indicated that the phenomenological classification of correlation states into intrinsic and dynamic correlations is very useful in understanding the complexity of 
electron-electron interactions. Recent studies on molecular s!stems [2:-31] hawe also shown that the Becker-Shirley classitication is feasible. This phemomenolug. ical classification allows for better communicattion between cexperimemtalists and bet ween experiment alists and theoreticians. who are necessarily working together to further the understanding of correlation mechanisms and the peaks they produce in photoelectron spectra.

\subsection{Ethylene}

Ethylene $\left[\mathrm{C}_{2} \mathrm{H}_{4}\left(D_{2 h}\right)\right]$ has the following electronic configuration in the groumd state: $\left(1 a_{g}\right)^{2}\left(1 b_{1 u}\right)^{2}\left(2 a_{g}\right)^{2}\left(2 b_{1 u}\right)^{2}\left(1 b_{2 u}\right)^{2}\left(3 a_{g}\right)^{2}\left(1 b_{3 g}\right)^{2}\left(1 b_{3 u}\right)^{2}$, having ${ }^{1} A_{y}$ symmetry. All orbital symmetries are referenced to the molecule lying in the $y z$ plane, with the $C-C$ double bond along the $z$ axis. Figure I shows the molecular geometry and the molecular orbitals of the ethylene molecule. Part (b) of Figure 1 shows the simple valence bonding molecular orbitals of ethylene, including the L(TMO) (lowest unoccupied molecular orbital) $1 b_{2 g}$ which corresponds to a $\pi^{*}$ antibonding orbital. The molecular orbitals can be considered to be composed of hydrogen $1 s$ and carbon $2 s$ and $2 p$ components in the simplest possible approxination. The low-lying carbon $1 s$ (binding energy of approximately $285 \mathrm{cV}$ ) orbitals are not considered to rarticipate in bonding. Filled and unfilled lobes are used to distinguish between orbitals of opposite phase. The $1 b_{2 g}\left(\pi^{*}\right)$ and $1 b_{s u}(\pi)$ orljtals are composed of the carbon $2 p_{x}$ orbitals which sit above and below the molecular $y z$ plane. The $1 b_{3 g}$ and $1 b_{2 u}$ orbitals are bonding $\left(1 b_{3 g}\right)$ and antibonding $\left(1 b_{2 u}\right)$ 
combinations of $\left({ }^{2} y_{y}\right.$ and $/ / \mathrm{l}$... Here, the $\left(\mathrm{C}_{2}\right.$ groups act as units which engage in $\pi$-like bonding. The $3 a_{y}$ ortital is a $\sigma$ bond composed of $\left(2 p_{z}\right.$ orbitals, lying along the major axis of the molerule. Because there are two carbon atoms, there are two inner valence orbitals, $2 b_{1 u}$ and $2 a_{y}$, predominantly composed of carbon 2.s orbitals, which behave in a $\sigma$-like manner, both bonding $\left(2 a_{g}\right)$ and antibonding $\left(2 b_{1 u}\right)$. The carbon $1 s$ orbitals corresponding to $l a_{g}$ and $l b_{1 u}$ (both considered to be nonbonding) are not shown.

The photoelectron spectrum (PES) of ethylene, the simplest unsaturated hydion arbon, has been investigated extensively both experimentally $[14,29-31.35$ 46] and theoretically [47-6:3] since the 1950's. The PES of the carbon $2 s$ region of ethylene was first reported by Gelius [14] using Al $K_{\alpha} \mathrm{X}$-rays (1487 $\mathrm{eV}$ photon energy) in 1974. It was also reported by Berndtsson et al [42] in 1975 and subsequently studied by Banna and Shirley [43] with $M g K_{\alpha}$ (1253 $\mathrm{eV}$ photon energy) and $Y . M_{\zeta}(132 \mathrm{eV}$ photon energy) X-ray sources. The peaks at $23.7 \mathrm{eV}$ and 19.2 $\mathrm{eV}$ binding energy were assigned as $2 a_{g}^{-1}$ and $2 b_{1 u}^{-1}$ primary ionization peaks, respectively, by Gelius [14]. The strong satellite (or "extra" peak) at $27.4 \mathrm{eV}$ in the $\mathrm{Mg} K_{\alpha}$ spectrum [43], with an intensity of $39 \%$ of the $2 a_{g}^{-1}$ primary peak, was originally assigned as a ${ }^{2} B_{1 u}$ correlation state $[14,42,43]$. The task of the earlier theoretical calculations [47-5i] was to assign the five main bands (below $20 \mathrm{cl}$ binding energy') through Koopmans Theorem [66] at the Hartree-Fock (HF) level. The first study that went beyond the HF approximation (Green's Function 


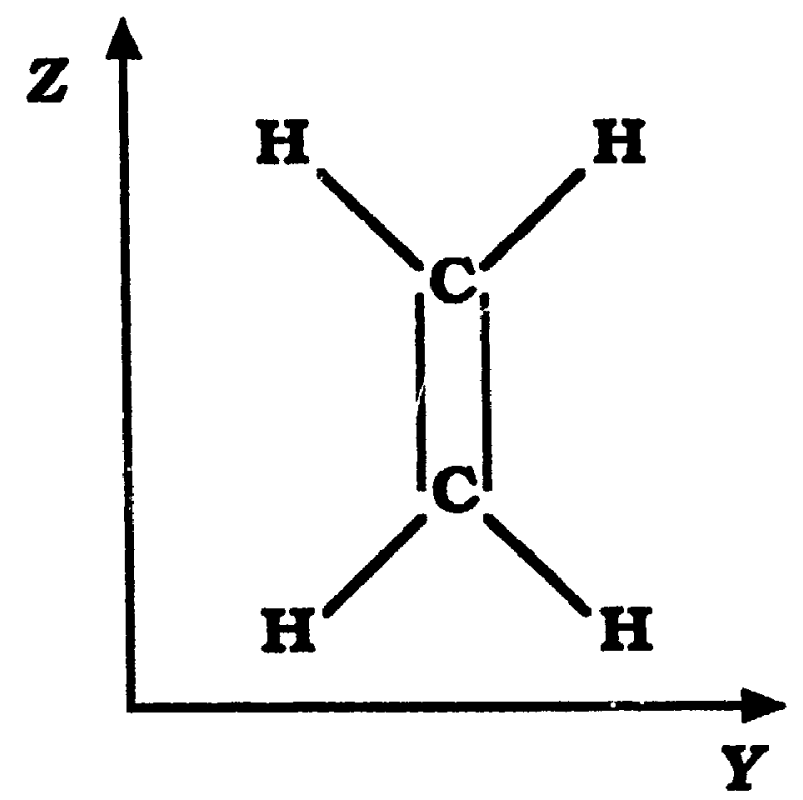

(a)

Figure 1: (a) The molecular geometry and (b) the molecular orbitals of the ethylene molecule. Filled and unfilled lobes are used to distinguish between orbitals of opposite phase. 
$1 b_{2 g}\left(\pi^{*}\right) \quad 1 b_{3 u}\left(\pi_{c=c}\right) \quad 1 b_{3 g}\left(\pi_{C H 2}\right) \quad 3 a_{g}\left(\sigma_{c-c}\right)$
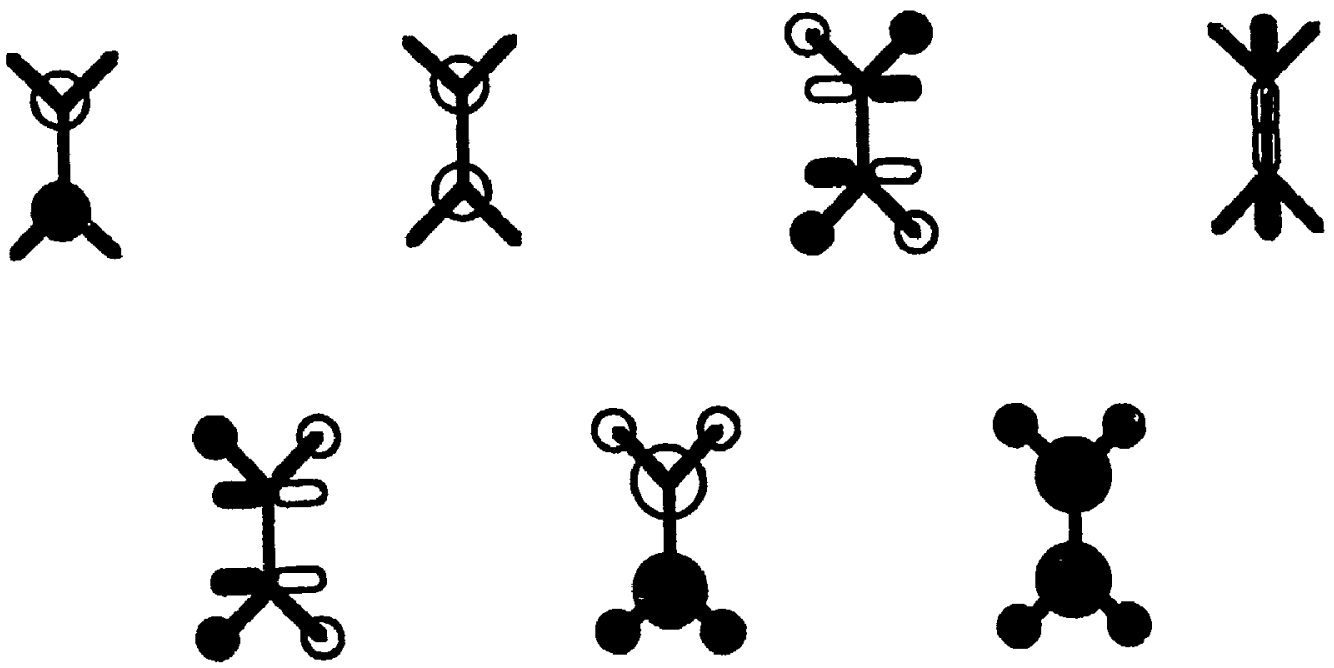

$1 b_{2 u}\left(\pi_{C H 2}\right) \quad 2 b_{1 u}\left(C_{2 s}{ }^{*}\right) \quad 2 a_{g}\left(C_{2 s}\right)$

(b) 
method) was published in 1976 [3i]. Martin and Davidson [ï9] performed a small configuration interaction ('1) calculation on the ethylene cation which agreed with experimental results. but indicated that the intense satellite belonged to the ${ }^{2} A_{y}$ symmetry manifold, with $30 \%$ of the intensity of the primary $2 a_{g}^{-1}$ peak. Sulsitquent Electron Momentum Spectroscopy (EMS) experiments [67,68\}, also known as binary $(e, 2 e)$ spectroscopy, confirmed this assignment; however. the intensity ratios obtained were different. Symmetry assignments in EMS experiments are made by comparing the momentum distribution of the correlation peak to the momentum distribution of the main peak. The shape of the momentum distribution for the correlation peak should match the shape of the momentum distribution of its parent peak. Differences in the correlation peak/main peak intensity ratios between EMS and PES have previously been observed in argon 3,. ionization spectra [23]. Investigation into the nature of these differences are actively being pursued $[69-72]$.

Cederbaum et al [61] performed Green's Function calculations, including more correlation configurations in their investigation than Martin and Davidson, but used smaller basis sets without diffuse Rydberg functions. The results agreved qualitatively with experiment, but suggested that there was a much richer st.ructure than could be resolved. Two ${ }^{2} A_{g}$ peaks (correlation states) of roughly equal intensity less than $1 \mathrm{eV}$ apart centered at $23.5 \mathrm{eV}$ and another two closely spared ${ }^{2} A_{g}$ peaks centered at $27.5 \mathrm{eV}$ were found. The intensity of this dual satellite 
$\left(27.5, V^{\prime}\right)$ was found to be $2.1 \%$ of the dual primary peak $\left(23.5,5 I^{\prime}\right)$. The recent MRSI)(I calculation of Murray and Davidson [60] lead to a similar conclusion as the work of Martin and Davidson [5!)], as neither calculation produced the twinning phenomenon of the ${ }^{2} A_{g}$ peak at $23.5 \mathrm{cV}$ binding energy or the satellite peak at $27.5 \mathrm{eV}$.

In addition to these early theoretical successes, Baker investigated the PES of ethylene using the EOM/propagator technique [62]. Two ${ }^{2} A_{g}$ peaks of roughly equal intensity centered at $23.2 \mathrm{eV}$ were found, but only one ${ }^{2} A_{g}$ satellite peak at $27.26 \mathrm{eV}$. Truncation of the virtual space caused the twinning phenomenon to disappear. Two calculations based on the Symmetry-Adapted-Cluster (SAC) expansion CI theory performed by Nakatsuji [63] and Wasada and Hirao [64] are also of interest as no twinning of the ${ }^{2} A_{g}$ primary peak was observed, nor was there any strong ${ }^{2} A_{g}$ satellite between 27.0 and $30.0 \mathrm{eV}$ binding energy. The $27.4 \mathrm{eV}$ satellite peak was assigned as $a^{2} B_{1 u}$ symmetry manifold correlation despite the earlier assignments to ${ }^{2} A_{g}$.

In this thesis, the well-known correlation peak at $27.4 \mathrm{eV}$ binding energy, belonging to the ${ }^{2} A_{g}$ symmetry manifold [29,31], has been confirmed as an intrinsic correlation as the ratio of satellite/main peak has been found to be effectively constant over a large photon energy range. Also, evidence of the dynamic nature of the newly discovered correlation peak at $21.4 \mathrm{eV}[30,31]$ has been obtained as the ratio of new correlation peak/main peak has a definite photon energy dependence. 


\section{Experimental}

The synchrotron photoelectron spectra (SPES) were obtained at the ('anadian Synchrot ron Radiation Facility (CSRF) at the Aladdin Storage Ring of the I'niversity of Wisconsin at Madison's Syuchrotron Radiation Center (SR(') in May 1991, August 1991, May 1992 and May 1993. The spectra were collected using the McPherson photoelectron spectrometer equipped with a channeltron detertor and a multichannel plate detector [73] in conjunction with the grasshopier grazing incidence monochromator [it] with high and low energy gratings. Figure 2 shows the basic schenatic of the McPherson photoelectron spectrometer. The synchrotron beam propagates perpendicularly to the plane of the diagram and is indicated by the small dark rectangle. Interactions between the photons and the gas sample occur in the gas cell. The photoelectrons are taken off at the pseudomagic angle appropriate for this equipment, $55.8^{\circ}$ with respect to the polarization axis [4]. The electron energy analyzer is a spherical analyzer with a mean radius of $36 \mathrm{~cm}$ in which a tunable guiding electric field is present to drive the photoelectrons towards the detector. Two detectors were used. In 1991, a Galileo Electro-Optics channel electron multiplier (channeltron) with a bias voltage of $2400 \mathrm{~V}$ and a gain of $1.2 \times 10^{8}$ was used. For the experiments conducted in 1992 and 1993, a Quantar open face multichannel plate position sensitive detector with a bias voltage of $3860 \mathrm{~V}$ and a gain of $5 \times 10^{7}$ was used. Extensive calibration studies of the multichannel plate were conducted by Zhifeng Liu using the 
$X_{10} 5$, and td peaks [7:3]. The grasshopper grazing incidence monochromator is so-named because its physical appearance reminds one of a grasshopper and the source beam grazes the first focussing mirror at an angle of only $1^{\circ}$. The beam is then focussed onto a nirror-slit combination that passes the beam onto a grating. which forusses the beam through the exit slit. Depending on the grating being used, the monochromator can deliver photons in the energy range 20 to $1200 \mathrm{eV}$. Two gratings were used in the experimentation. A grating with 600 grooves $/ \mathrm{mm}$ provided photons in the photon energy range 30 to $70 \mathrm{eV}$. A second grating, with 1800 grooves $/ \mathrm{mm}$, provided photons in the range 70 to $220 \mathrm{eV}$. Typical slit widths used were 30 to $50 \mu \mathrm{m}$. Figure 3 shows the Aladdin Storage Ring. The Canadian Sychrotron Radiation Facility is located at bending magnet 09 (where synchrotron radiation comes off tangentially to the electron flow) at the bottom of the diagram. The numbers 1,2 and 3 refer to the ports (beamlines) on that bending magnet. The grasshopper's port designation is then 091. Spectra were also obtained by Dr. Bawagan in December 1992 with the 3 meter toroidal grating monochromator (3m-TGM) (port 012) [75]. In a TGM, a toroidal grating is used to focus the photon beam between the entrance and exit slits. Ellipsoidal mirrors are used to focus the beam horizontally on the exit slit. Angular corrections to the differential cross sections are not required as the photoelectrons were collected at the pseudomagic angle [4]. All reported intensity ratios are corrected for analyzer transmission effects [27]. Transmission corrections are zecessary as 
the analyzer is biased towards higher kinetic encrgy photoclectrons; slow moving photoelectrons are easily lost because of the loug thight time through the redatively. large estergy analyzer. Further back pround corrections were made for low energy scattered electrons and stray light for the data obtained with the :m-T(iM. A single Matheson Gas Products research purity (99.991\%) sample of ethylene was used for all experimentation. Further experintents were also conducted with isotopically labelled ethylene $\left(1-{ }^{13} \mathrm{C}\right.$-ethylene) from Isotec $(99 \%$ pure $)$. This labelled ethylene has one ${ }^{13} \mathrm{C}$ isotope - the other carbon atom is "normal" ${ }^{12} \mathrm{C}$ ".

High vacuum in the McPherson spectrometer is maintained throngh an extensive pumping systen. The anatyzer chanber and gas cell chamber are pumperd separately with Balzers turbomolecular pumps (models TPV 510 and 330, rospectively). The entire system is rough pumped and backed by a Balzers rotary pump (DUO 008D). The gas inlet system is pumped by a small 'TRI VA(: turbu pump. Baseline pressure in the analyzer chamber is typically $10^{-7}$ torr or lower. When sample is introduced, the gas cell chamber pressure increases to typically $5 \times 10^{-5}$ torr. The actual pressure in the gas cell is estimated to be two orders of magnitude higher.

The transmission of the spectrometer has been thoroughly checked using neon $2 s$ and $2 p$ ionization cross sections for calibration studies. The intensity ratio of the $\mathrm{Ne} 2 s$ peak to the $2 p$ peak is studied as a function of kinetic energy. The transmission function of the spectrometer can then be obtained by comparing 


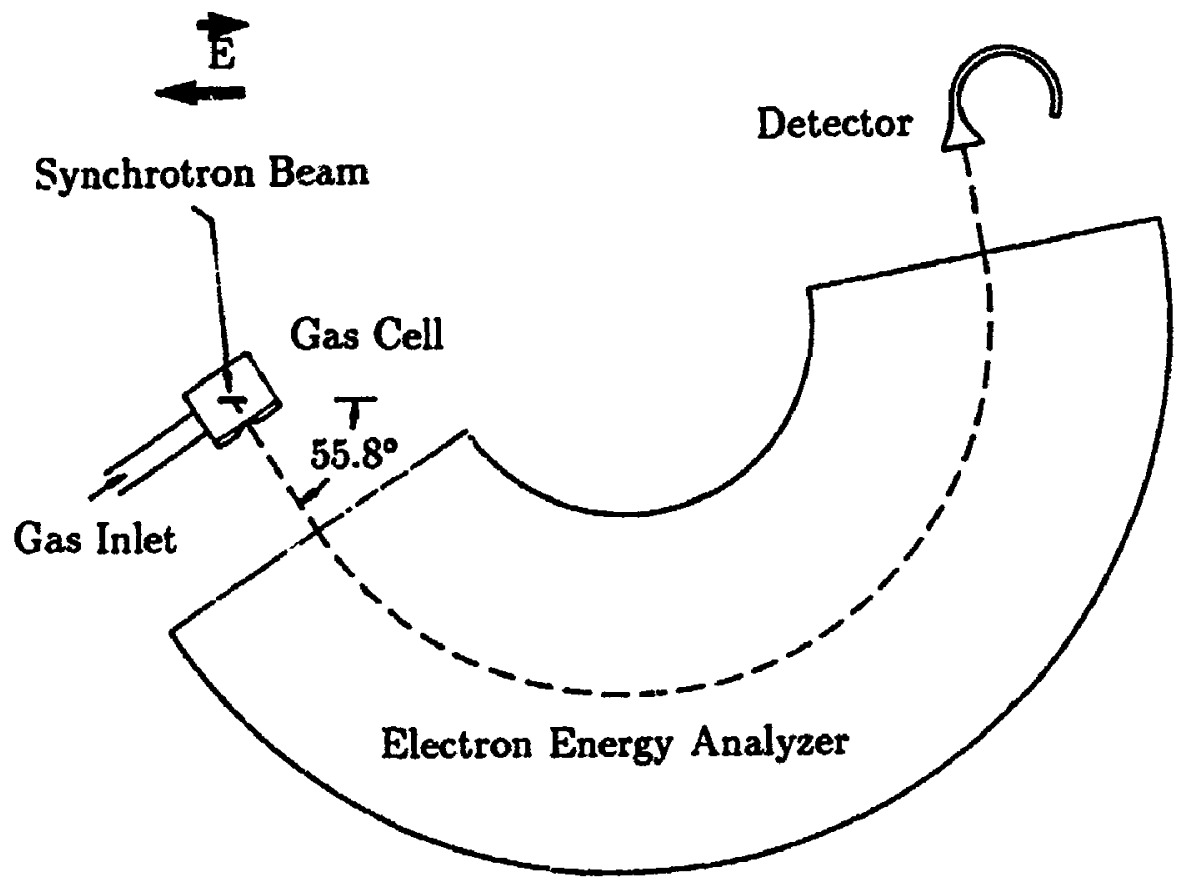

Figure 2: Schematic of the McPherson photoelectron spectrometer. The synchrotron beam is perpendicular to the plane of the diagram. The analyzer has a mean radius of $36 \mathrm{~cm}$. The dashed line represents the path of the photoelectrons through the analyzer. The diagram is not to scale. Adapted from reference 4. 


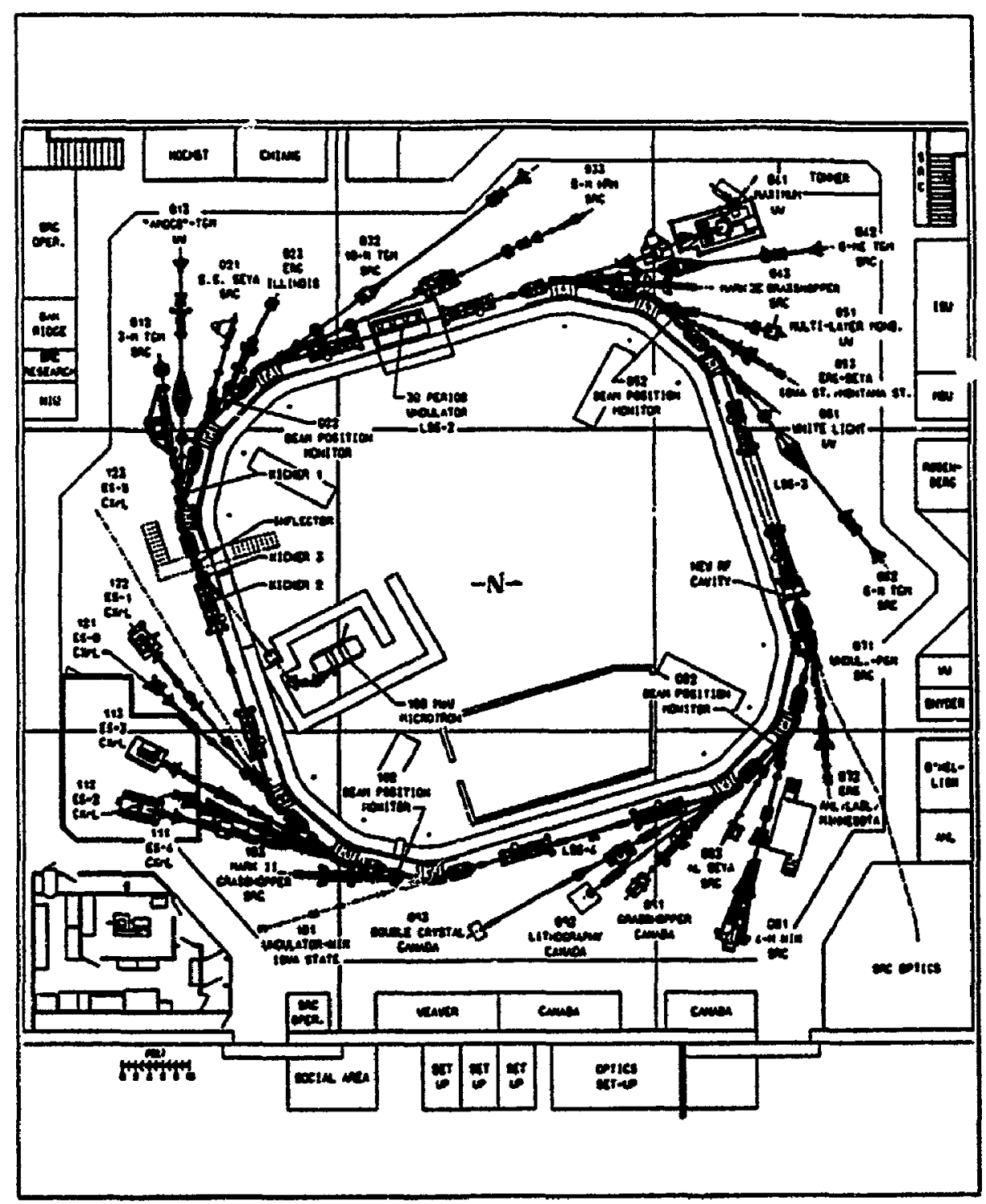

Figure 3: The Aladdin Storage Ring at the University of Wisconsin at Madison. The Canadian Synchrotron Radiation Facility is located at the bottom of the diagram, at ports 091-093. Adapted from reference 76. 
the $2 . s / 2 p$ ratio with literature. In general. the intensity or area of a peak $(A)$ in the photoelectron spectra obtained with this analyzer will be given by:

$$
A=\text { constant } * \sigma(h \nu) * I_{0}(h \nu) * T\left(E_{\kappa}\right)
$$

where $\sigma(h \nu)$ is the cross section for the photoionization, $I_{0}(h \nu)$ is the photon intensity at photon energy $h \nu$ and $T\left(E_{K}\right)$ is the analyzer transmission function at the photoelectron kinetic energy $E_{k}$. Spectra are obtained rapidly (typically less than five minutes) and so $I_{0}(h \nu)$ is effectively constant over a scan of the spectrum. And so:

$$
A \approx \text { constant } * \sigma(h \nu) * T\left(E_{K}\right)
$$

The intensity (area) ratios of the Ne $2 s$ to $2 p$ peaks is calculated and compared with the literature values of the photoionization cross section, allowing $T\left(E_{K^{*}}\right)$ to be determined. It was confirmed that over the range that the SPES studies were conducted, the transmission function is essentially linear in kinetic energy, or:

$$
T\left(E_{K}\right)=\text { constant } * E_{K}
$$

as previous studies with the analyzer have indicated [27]. Intensity ratios of photoelectron peaks are corrected for transmission effects; these corrections range from approximately $20 \%$ at $45 \mathrm{eV}$ down to approximately $2 \%$ at $200 \mathrm{eV}$.

Monochromators function as wavelength filters by limiting the photons that pass to specific wavelengths, ie those that complete their wavecycle in the distance between the focussing elements. However, energies that are integer multiples of the 
selected photon energy will also pass through because the ir wavelengt hs are integer divisors of the selected wavelength. These radiations are referred to as higher order radiations. For example, if the monochromator is set for $h l^{\prime}$, some second order radiation, $2 h \nu$ in energy, will pass through. This radiation, depending on its intensity and the sample being studied, can interfere in the experiment. The intensity of second order radiations from the grasshopper monochromator was also investigated via the $2 p$ peak of neon. The second order radiations were stuctied by setting the monochromator for a certain photon energy, $h \nu$, and then scanning the photoelectron kinetic energy range that would be appropriate for $2 h u$. 'The' transmission corrected intensity ratio of the second order $2 p$ peak to the first orcler $2 p$ peak was then calculated as a function of photon energy. It was found that se 'ond order radiation is of significant intensity only at energies lower than those over which the vast majority of spectra were obtained. The second order radiation peaks in intensity (approximately $40 \%$ ) between 30 and $3.5 \mathrm{eV}$ primary photon energy, but quickly falls off, down to approximately $1 \%$ of the first order intensity by $45 \mathrm{eV}$. A Table and Figure of the second order data appear in Appendix $\mathrm{A}$. There is, therefore, no interference from second order radiation.

The total experimental energy resolution, including both electron analyzer and monochromator effects, was found to range from $490 \mathrm{meV}$ fwhm at $45 \mathrm{eV}$ to $820 \mathrm{meV}$ fwhm at $200 \mathrm{eV}$. The total experimental resolution is given by [4]:

$$
\Gamma_{\text {total }}^{2} \approx \Gamma_{\text {analyzer }}^{2}+\Gamma_{\text {monochromator }}^{2}+\Gamma_{\text {natural width }}^{2}
$$


The total resolution of the experiments is limited by that of the monochromator. The natural widths of peaks are, of course, beyond experimental control. The energy resolution of the analyzer is good, approximately $40 \mathrm{meV}$ fuhm [4], and it is relatively stable over the range of energies studied. The energy resolution of the grasshopper, however, becomes poorer with increasing photon energy and slit width. At higher energies, the photon flux from the grasshopper monochromator will be lower, and so the entrance and exit slits have to be opened wider to increase the flux of photons coming out of the monochromator and hence to increase the observed count rate at the detector. Opening the slits wider causes the distribution of energy $(h \nu \pm \delta)$ that the grasshopper passes to increase (ie $\delta$ increases as the slits are opened wider) and so, the result is poorer energy resolution. Also, for a fixed slit width, the energy resolution becomes poorer with increasing photon energy.

In May of 1991, the first ethylene spectra were run in the photon energy range 70 to $220 \mathrm{eV}$. Later that summer, in August, the first $1{ }^{13} \mathrm{C}$-ethylene spectra were taken, in the same energy range. On analysis of these spectra, it was found that the major correlation (satellite) peak at $27.4 \mathrm{eV}$ binding energy was intrinsic in nature, according to the Becker-Shirley scheme [5], as the ratio with respect to its parent was constant [29]. Comparison with theoreicical calculations was also very successful [29]. In May of 1992, lower energy spectra in the photon energy range of 30 to $70 \mathrm{eV}$ of both species were run to investigate the ratio of the major satellite 
to the $2 a_{g}^{-1}$ main peak closer to its threshold of $27.4 \cdot 1$. On analysis of these spectra, the new correlation peak at 21.4 a $l^{\circ}$ binding energy was discovered. It had been overlooked in the previous spect ra because of its photon energy dependence . the peak has very little intensity above $70 \mathrm{el}$ photon energy. Also at this time, the second order radiation study with Ne was conducted, to check for interferences. Later that year, in December, Dr. Bawagan ran the spectra of both speciess on the $3 m$-TGM at the lower energy range to check whether the observed spectral features were independent of the type of monochromator. Finally, in May of 1993, the last spectra of normal ethylene were run at the higher energy range to investigate in detail the decrease in intensity of the new (minor) correlation peak. Also, a pressure study was conducted with ethylene to investigate any possible contribution from inelastic scattering processes. During all beam runs, the transmission of the spectrometer was checked with Ne. 


\section{Analysis}

Spectra obtained from the instrument are a binary format. To ease the analysis, the spectra are first converted to ASC'II. ('urve fitting is done on computer, using the program PEAKFIT (Jandel Scientific). Gaussian peaks are used. For the $2 b_{1 u}^{-1}$, $2 a_{g}^{-1}$ and major satellite peaks, vibrational broadening made it necessary to use two gaussians for each peak to ensure complete coverage of the area under the peaks. For spectra obtained with the grasshopper monochromator, backgrounds were fitted with straight lines.

For the spectra obtained with the $3 \mathrm{~m}$-TGM, the low energy scattering of electrons caused an exponential-decay-like low kinetic energy background which made a further correction necessary. "No gas" background spectra were run in conjunction with the ethylene spectra at the various photon energies; these background spectra were then scaled (all points multiplied by a constant) to match the low kinetic energy tail of the ethylene spectra. The background spectra were then subtracted from the ethylene spectra and the resulting background-corrected spectra were analyzed as the other (grasshopper) spectra.

After the curve fit is acceptable, the various intensity ratios are calculated by comparing the areas under the specific peaks. Transmission corrections are done by dividing the area of a peak by the kinetic energy of its mean.

The interesting photon energy dependence of the ratio of the new correlation peak to the $2 b_{1 u}^{-1}$ main peak was investigated in detail. The data was curve fit 
using PEAkFIT with an exponential decay, a constant, live gaussian peaks and a damped sine wave function to obtain an approxinate functional form. The purely. oscillatory component (the gaussians and the damped sine) was extrarted and Fast Fourier Transformed using gRaftool (sec Results section for devails).

In order to compare the experimental spectra with theoretical calculations, a simple FORTRAN program was written to convolute the theoretical pole strengthis with gaussian peaks whose widths are consistent with those observed experimentally. Since the photoionization dipole matrix elements are not included in the theoretical calulations, proper comparisons between experiment and theory can only be made across the same symmetry mainifold. 


\section{Results and Discussion}

\subsection{The Intrinsic Correlation State}

Figure 4 shows a sample synch:otron photoelectron spectrum of normal ethylene, taken at $91.1 \mathrm{eV}$ photon eilergy, having peaks associated with ionization from the six valence molecular orbitals. The extra peak, labelled "sat", is the well-known correlation (satellite) peak originally observed by Gelius [14]. Also shown is the general curve fitting form - gaussian peaks and a linear background. Figure 5 shows a similar spectrum, taken at $59.5 \mathrm{el}$.

Figure 6 shows a sample spectrum of ${ }^{13} \mathrm{C}$-labelled ethylene $\left(1-{ }^{13} \mathrm{C}-\mathrm{C}_{2} \mathrm{H}_{4}\right)$ at $59.5 \mathrm{eV}$ photon energy. It can be seen that the SPES spectrum of isotopically labelled ethylene (Fig. 6) is very similar to that of normal ethylene (Fig. 5) at the same photon energy with regards to relative intensities, binding energies and peak widths, further confirming that the extra structure (labelled "sat") found in the SPES is of "electronic origins", ie largely independent of nuclear structure. It is well-known that vibrational frequencies, and thus the Franck-Condon widths associated with photoionization, are influenced by deuterium substitution of the hydrogen atoms in ethylene [77]. In this particular study, ${ }^{13} \mathrm{C}$ substitution is not expected to change significantly the vibrational frequencies of ethylene (as well as Franck-Condon widths) for the inner valence orbitals, however it is a reasonably sensitive lest of "nuclear effects" in correlation states. ${ }^{13} \mathrm{C}$ substitution introduces an asymmetric $1 / 12$ change in nuclear mass of one of the carbons. If these extra 


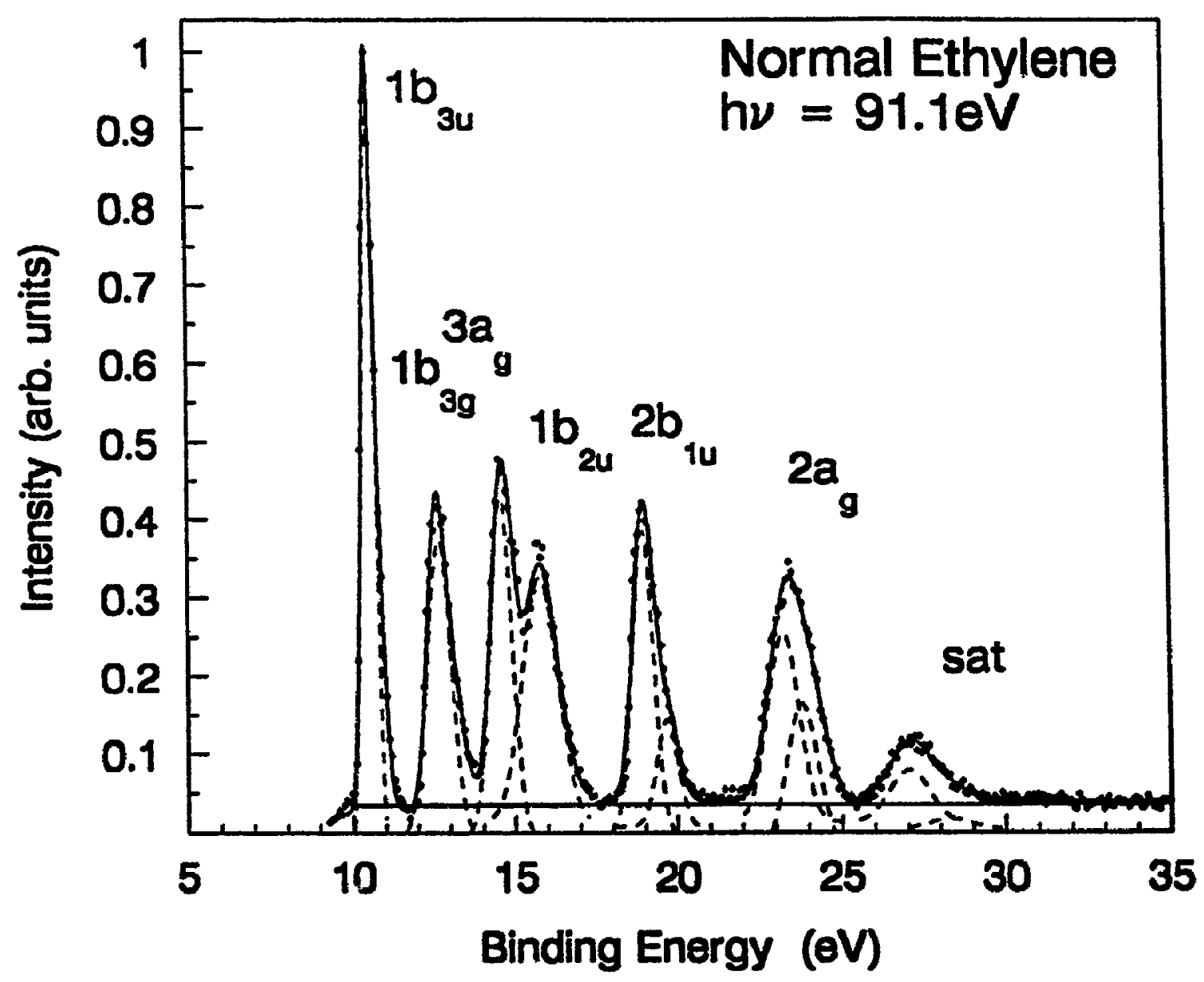

Figure 4: Synchrotron PES spectrum of normal ethylene at $91.1 \mathrm{eV}$. The curvefitting model used for all of the spectra is shown by the dashed gaussians and the solid baseline. Single gaussian peaks are fitted to the experimental peaks attributed to ionization from the outer valence orbitals. Two gaussian peaks of equal widths are fitted to each of the experimental peaks attributed to the satellite $(27.4 \mathrm{eV}), 2 a_{g}^{-1}$ and $1 b_{14}^{-1}$. The sum of the peaks is represented as a solid line. The spectrum as shown is not corrected for transmission effects. The estimated total experimental energy resolution is $470 \mathrm{meV}$ fwhm. The standard orbital notation assumes $D_{2 h}$ symmetry with the molecule in the $y z$ plane (see Figure 1). 


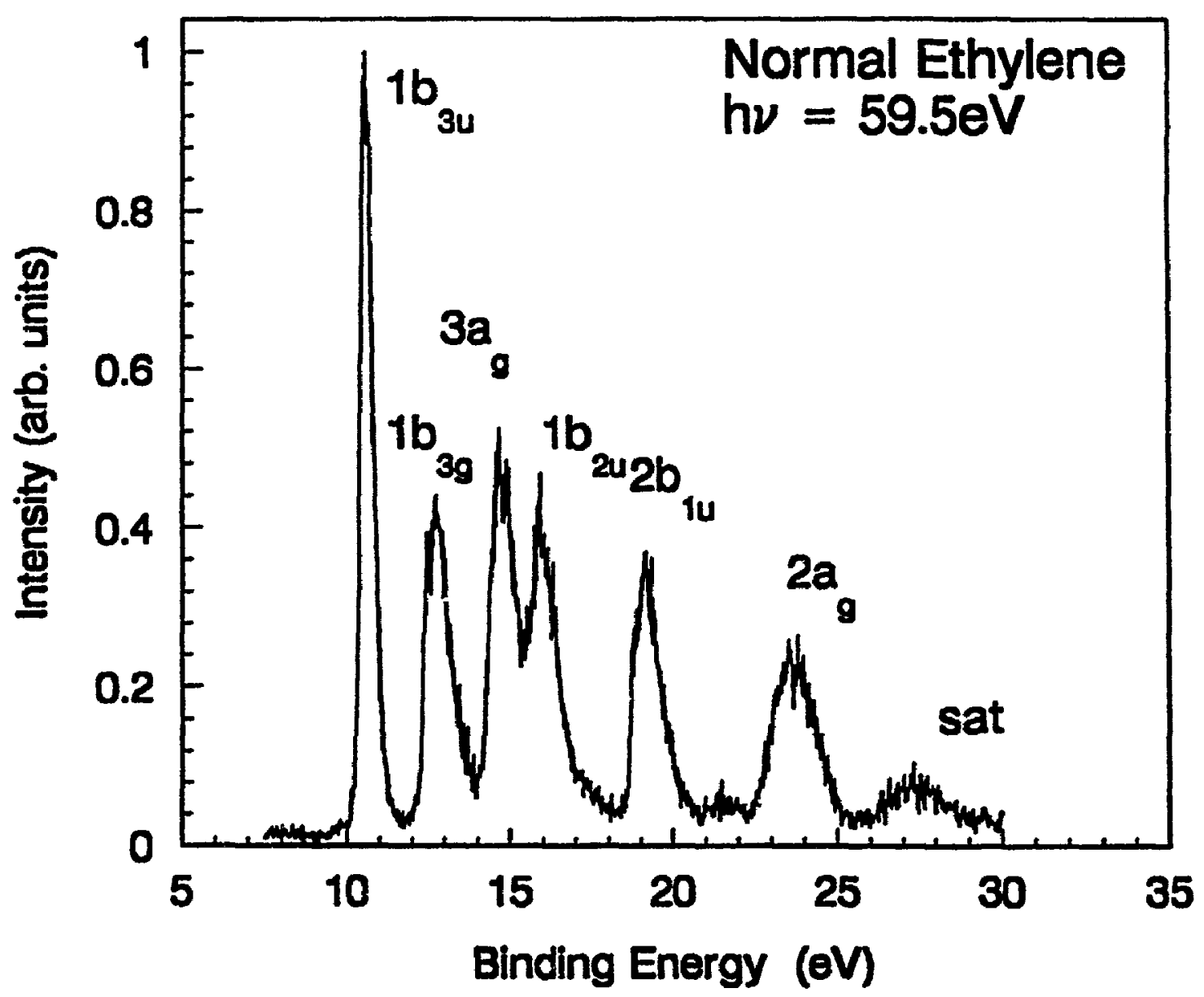

Figure 5: SPES of normal ethylene taken at $59.5 \mathrm{eV}$. The spectrum as shown is not corrected for transmission effects. 


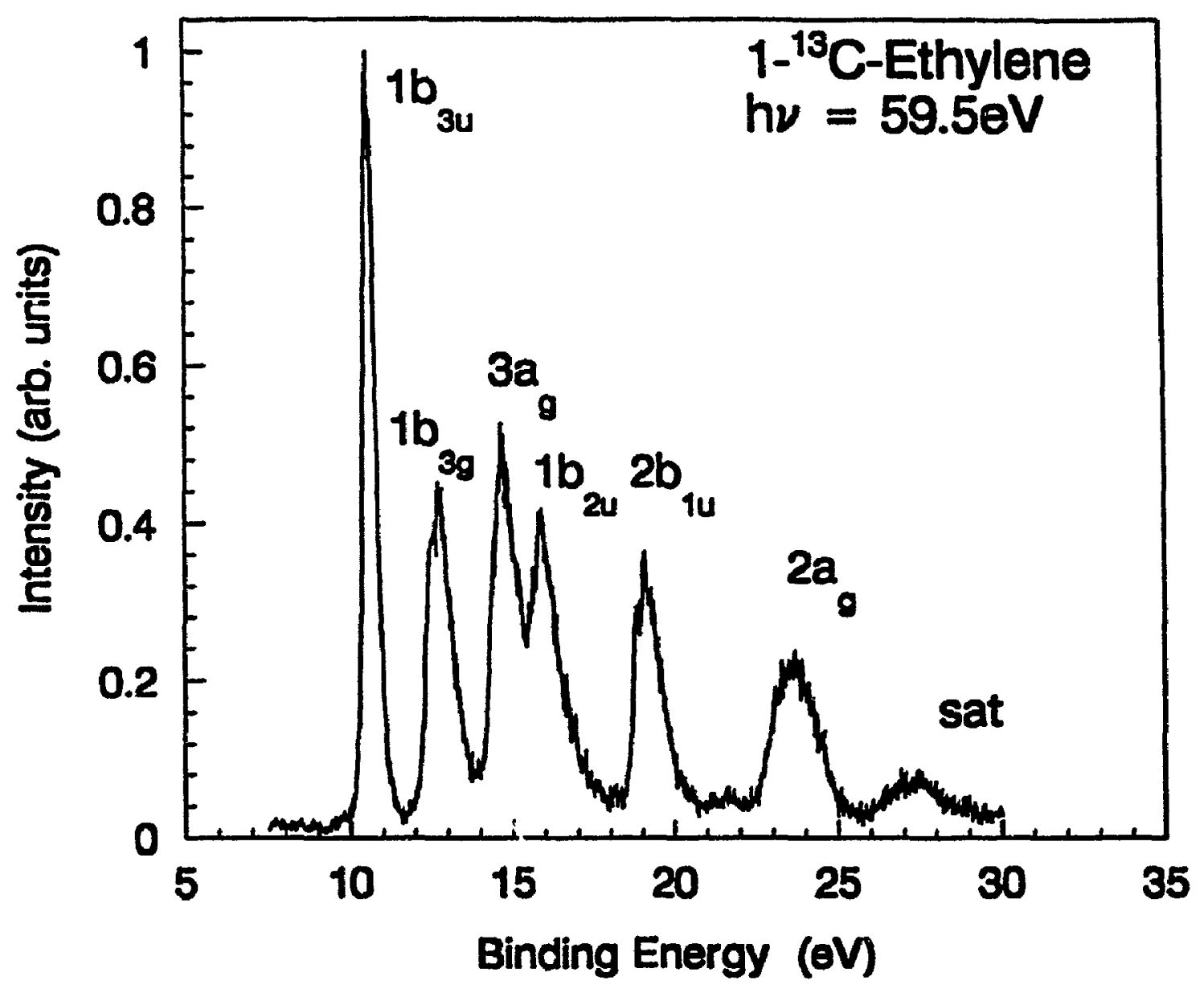

Figure 6: SPES of $1^{13} \mathrm{C}$-ethylene taken at $59.5 \mathrm{eV}$. The spectrum as shown is not corrected for transmission effects. The isotopically labelled ethylene is seen to have a similar spectrum to that of normal ethylene (see Figure 5). 
peaks are caused by sume electron-nuclear interaction, then there should be some differences in their inner valence SPES. No difference is observed (cf Fig. 5 and 6). and thus "nuclear effects" as a possible explanation for the satellite structure in the spectrum of ethylesie is discomited. A percent difference spectrum for the two species at $59.5 \mathrm{eV}$ is given in Appendix B. It can be seen that in the binding energy range of interest, 18 to $30 \mathrm{EV}$, there are no truly discernible features, merely noise. confirming that the spectra of normal and labelled ethylene are very similar.

The correlation peak at $2 \pi .4 \mathrm{eV}$ has been thoroughly investigated by measuring the satellite/uain $2 a_{y}^{-1}$ peak intensity ratio at different photon energies from 37 to $223 \mathrm{eV}$. These ratios are presented in Table 1 and Figure 7, with previous X-ray measurements presented for comparison. The ratio is seen to be effectively constant over the entire energy range, even as the threshold $(27.4 \mathrm{eV})$ is approached $[29,31]$. SPES at photon energies less than $h \nu=36 \mathrm{eV}$ are difficult to obtain with the present electrostatic photoelectron energy analyzer. The difficulty arises from background problems associated with low energy scattered electrons. The dashed line (in both Figures 7 and 8 ) represents the ratio calculated from the MRSDCI-ANO calculation for normal ethylene [31]. The high value for Al $K_{\alpha}$ is probably a result of experimental factors unaccounted for (in this work) as the published spectrum was deconvoluted using a peak profile similar to that used for the fitting of the synchrotron spectra. Figure 8 shows the photon energy dependence of the correlation peak at $27.4 \mathrm{eV}$ in the case of $1 .{ }^{13} \mathrm{C}$-ethylene; the ratio 
Table 1: Transmission corrected satellite/main $2 a_{y}^{-1}$ intensity ratios for the intrinsic correlation state at $2 \bar{T} .1 \mathrm{el}$ ' binding energy of normal ethylenc.

\begin{tabular}{|c|c|c|c|c|c|}
\hline $\begin{array}{c}\text { Photon } \\
\text { Energy } \\
\text { (eV) }\end{array}$ & $\begin{array}{c}\text { Intensity } \\
\text { Ratio }^{a} \\
( \pm \sigma)\end{array}$ & $\begin{array}{c}\text { Photon } \\
\text { Energy } \\
\text { (eV) }\end{array}$ & $\begin{array}{c}\text { Intensity } \\
\text { Ratio'2 } \\
( \pm \sigma)\end{array}$ & $\begin{array}{l}\text { Photem } \\
\text { Encrgy } \\
\text { (rl) }\end{array}$ & $\begin{array}{c}\text { Intensity } \\
\text { R. }^{a} \omega^{a} \\
( \pm \sigma)\end{array}$ \\
\hline $36.7^{T}$ & $0.352(0.047)$ & $62.7^{T}$ & $0.336(0.035)$ & 136.5 & $0.35+(0.0 \cdot 2 \cdot 1)$ \\
\hline $37.8^{T}$ & $0.378(0.063)$ & 64.5 & $0.339(0.01 t)$ & $1+11.6$ & $0.367(0.02 \cdot 1)$ \\
\hline $38.7^{T}$ & $0.380(0.049)$ & $6.1 .7^{T}$ & $0.345(0.033)$ & 146.8 & $0.372(0.020)$ \\
\hline 39.5 & $0.361(0.031)$ & $66.7^{T}$ & $0.339(0.019)$ & 151.1 & $0.353(0.010)$ \\
\hline $39.7^{T}$ & $0.344(0.057)$ & $68.7^{T}$ & $0.352(0.025)$ & 151.9 & $0.36+(0.0) 30)$ \\
\hline $40.7^{T}$ & $0.323(0.042)$ & 69.5 & $0.352(0.010)$ & 157.0 & $0.417(0.025)$ \\
\hline $41.8^{T}$ & $0.337(0.033)$ & 70.6 & $0.360(0.030)$ & 162.1 & $0.113(0.0331)$ \\
\hline $42.7^{T}$ & $0.320(0.061)$ & $70.7^{T}$ & $0.350(0.046)$ & 167.1 & $0.398(0.023)$ \\
\hline $43.8^{T}$ & $0.362(0.031)$ & 71.1 & $0.324(0.075)$ & 171.2 & $0.32 \times 2(0.027)$ \\
\hline 44.5 & $0.359(0.016)$ & $72.7^{T}$ & $0.331(0.046)$ & 172.2 & $0.390(0.026)$ \\
\hline $44.7^{T}$ & $0.345(0.035)$ & 75.6 & $0.345(0.035)$ & 177.2 & $0.402(0.023)$ \\
\hline $46.7^{T}$ & $0.318(0.038)$ & 80.7 & $0.351(0.033)$ & 182.4 & $0.390(0.030)$ \\
\hline $46.8^{T}$ & $0.343(0.043)$ & 85.8 & $0.379\left(0.04^{\circ}\right)$ & 187.6 & $0.413(0.024)$ \\
\hline $48.7^{T}$ & $0.337(0.029)$ & 90.8 & $0.347(0.028)$ & 192.6 & $0.420(0.020)$ \\
\hline $48.8^{T}$ & $0.352(0.021)$ & 91.1 & $0.342(0.009)$ & 197.7 & $0.386(0.026)$ \\
\hline 49.5 & $0.345(0.010)$ & 95.9 & $0.361(0.040)$ & 201.1 & $0.341(0.036)$ \\
\hline $50.7^{T}$ & $0.323(0.031)$ & 101.1 & $0.373(0.028)$ & 202.7 & $0.350(0.030)$ \\
\hline $50.8^{T}$ & $0.360(0.063)$ & 101.2 & $0.327(0.039)$ & 207.8 & $0.392(0.020)$ \\
\hline $52.7^{T}$ & $0.320(0.025)$ & 106.1 & $0.348(0.029)$ & 212.9 & $0.391(0.025)$ \\
\hline $52.8^{T}$ & $0.304(0.026)$ & 111.2 & $0.350(0.026)$ & 217.8 & $0.379(0.024)$ \\
\hline 54.5 & $0.344(0.010)$ & 116.3 & $0.350(0.031)$ & 221.2 & $0.339(0.026)$ \\
\hline $54.7^{T}$ & $0.326(0.024)$ & 121.1 & $0.348(0.016)$ & 222.9 & $0.391(0.030)$ \\
\hline $56.7^{T}$ & $0.332(0.023)$ & 121.3 & $0.303(0.023)$ & $132^{b}$ & $0.2(0.4)$ \\
\hline $58.7^{T}$ & $0.336(0.035)$ & 126.4 & $0.330(0.025)$ & $1253^{b}$ & $0.39(0.06)$ \\
\hline 59.5 & $0.338(0.011)$ & 131.5 & $0.370(0.023)$ & $1487^{c}$ & $0.46(0.03)$ \\
\hline $60.7^{T}$ & $0.326(0.020)$ & & & & \\
\hline
\end{tabular}

auoted error limits refer to uncertainties from gaussian curve fitting aloue. Systematic errors due to the assumed baseline and transmission function are rstimated to be less than $5 \%$ for results obtained with the grasshopper and on the order of $10 \%$ for those obtained with the $3 \mathrm{~m}$-'TGM.

$T$ Results from the $3 \mathrm{~m}-\mathrm{TCM}$, all other values are from the grasshopper monochromator.

X-ray results: ${ }^{b}$ ref. $[43] .{ }^{c}$ ref. [42]. 


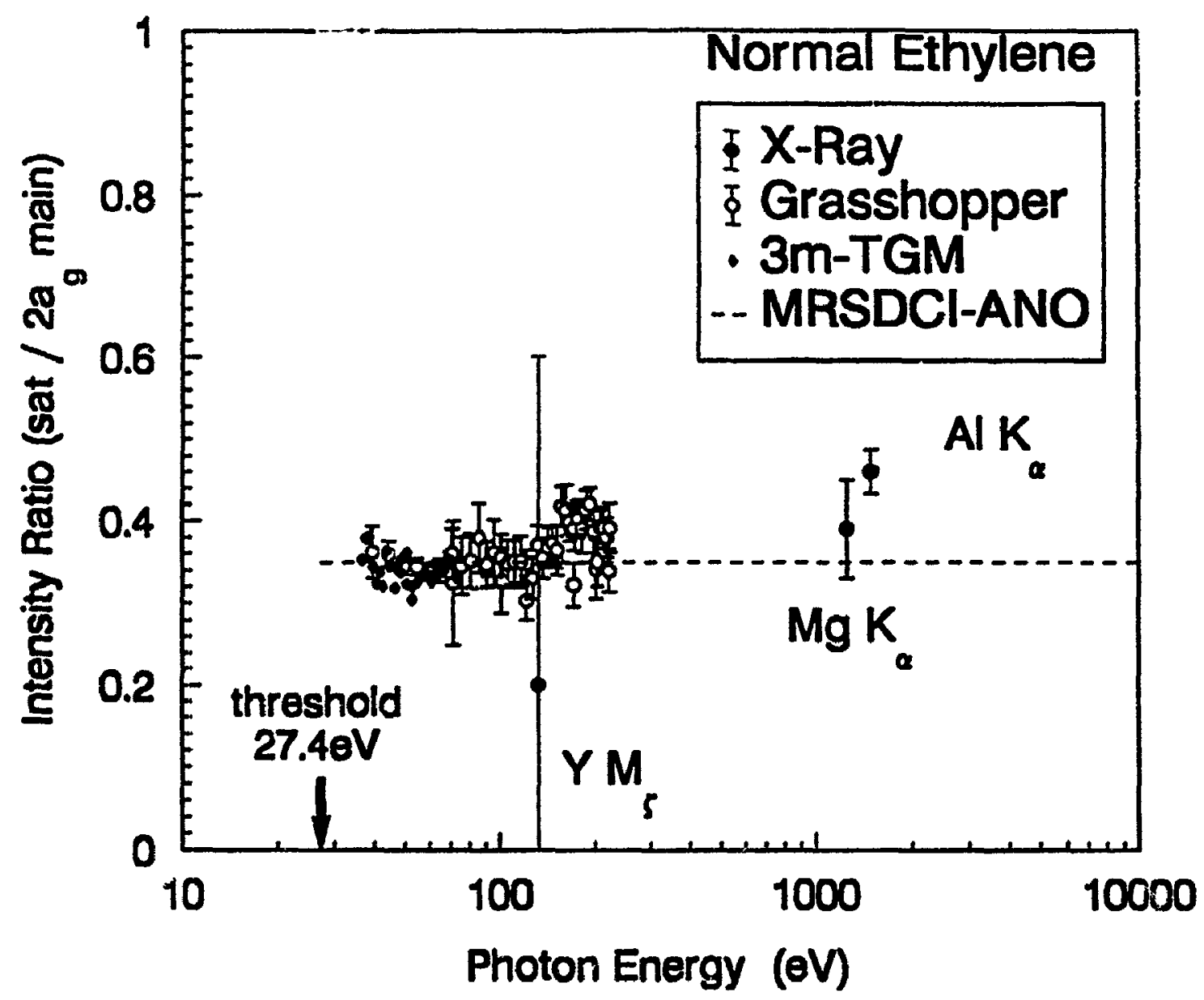

Figure 7: Photon energy dependence of the satellite/main $2 a_{g}^{-1}$ peak intensity ratios derived from synchrotron PES measurements for normal ethylene. The experimentally derived ratios (Table 1 ) are corrected for transmission effects. The open circles with error bars are data from the grasshopper monochromator and the solid diarisids are data from the $3 m$-TGM. Previous X-ray measurements $[42,43]$ are shown as solid circles. The dashed line is the predicted satellite/main intensity ratio from the MRSDCI-ANO calculation (see Table 7). 
Table 2: Transmission corrected satellite intensity ratios for $1{ }^{13}$ ('ethylene for the major correlation peu $x^{\prime}$ at $27.4 \mathrm{el}$ binding energy.

\begin{tabular}{|c|c|}
\hline $\begin{array}{c}\text { Photon Energy } \\
(\mathrm{eV})\end{array}$ & $\begin{array}{c}\text { Intensity Ratio }{ }^{a}( \pm \sigma) \\
\text { satellite/main } 2 a_{2}^{-1}\end{array}$ \\
\hline 44.5 & $0.329(0.029)$ \\
49.5 & $0.326(0.029)$ \\
& $0.366(0.060)$ \\
54.5 & $0.313(0.028)$ \\
$58.8^{T}$ & $0.313(0.054)$ \\
& $0.360(0.099)$ \\
& $0.250(0.084)$ \\
59.5 & $0.313(0.041)$ \\
& $0.299(0.023)$ \\
64.5 & $0.291(0.023)$ \\
69.5 & $0.293(0.023)$ \\
& $0.288(0.030)$ \\
91.1 & $0.330(0.042)$ \\
121.7 & $0.338(0.067)$ \\
151.9 & $0.315(0.094)$ \\
\hline
\end{tabular}

a Quoted error limits are statistical errors from gaussian curve fitting alone and do not include systematic errors. Systematic errors due to the assumed baseline and transmission function are estimated to be less than $5 \%$ for the results obtained with the grasshopper and on the order of $10 \%$ for those obtained with the $3 m-$ TGM.

$T$ The $58.8 \mathrm{eV}$ results are from the $3 m-T G M$, all other values are from the grasshopper monochromator. 


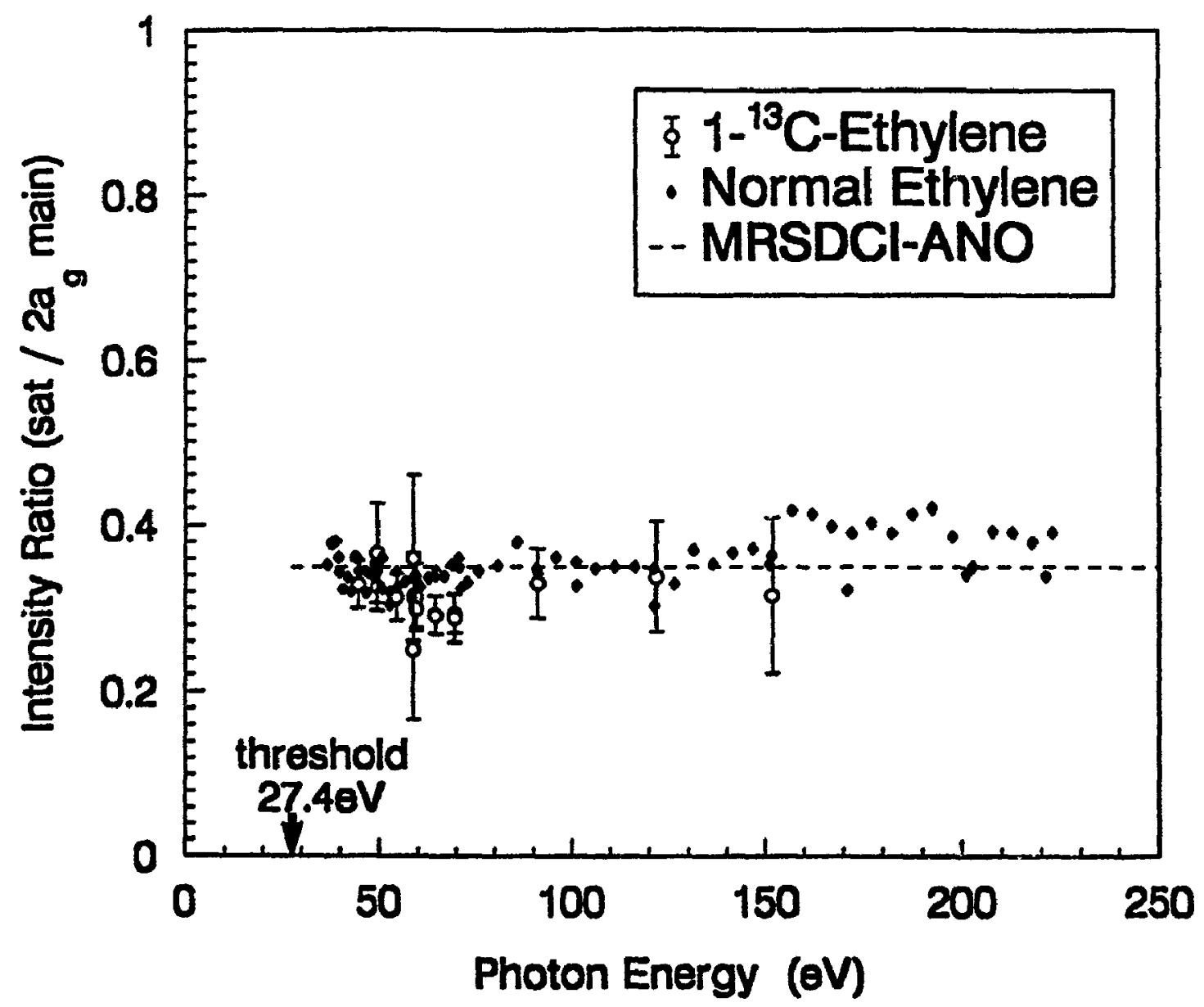

Figure 8: Photon energy dependence of the major satellite/main $2 a_{g}^{-1}$ peak intensity ratios for $1{ }^{13} \mathrm{C}$-ethylene. The ratios (Table 2) are corrected for transmission effects and shown as open circles with error bars. The ratios for normal ethylene are shown as solid diamonds for comparison. 
values are given in Table 2 . The constant value" of the intensity ratio observed in both normal (Fig. 7 ) and labelled (Fig. 8 ) ethylene is indicative of an "intrinsic" correlation, using the notation of Becker and Shirley [5], a result of initial and/or final state configuration interaction. These configuration interactions are always occuring, and the photoionization process only allows them to be seen - it does not produce them - thus, there should be no photon energy dependence. The threshold has been approached, within the limitations of the current instrumentation, yet the constant trend has been observed to continue, providing further evidence against contributions from mechanisms like shake-up or interchannel coupling [5]. The excellent quantitative agreement between experiment and theory (as shown by the dashed line in Fig. 7) indicates that the theoretical understanding of the ${ }^{2} A_{g}$ correlation peak at $27.4 \mathrm{eV}$ is on a solid foundation $[29,31]$.

\subsection{The Dynamic Correlation State}

A new correlation peak at (21.4 \pm 0.1$) \mathrm{eV}$ binding energy is observed in the synchrotron photoelectron spectrum of ethylene taken at $49.5 \mathrm{eV}$ photon energy (see Figure 9). The energy scale was calibrated by aligning the binding energy of the $1 b_{3 u}^{-1}$ peak $(10.51 \mathrm{eV})$ with high resolution He I spectra [78]. The same correlation peak can be observed in a previously reported spectrum [45], however it was not acknowledged in that particular study. A 1978 Green's function calculation by

*Careful examination of the data in the range 150 to $220 \mathrm{eV}$ indicates a slightly higher intensity ratio; however, it should be noted that the total experimental error (systematic + statistical) prevents any additional interpretations from being made. 


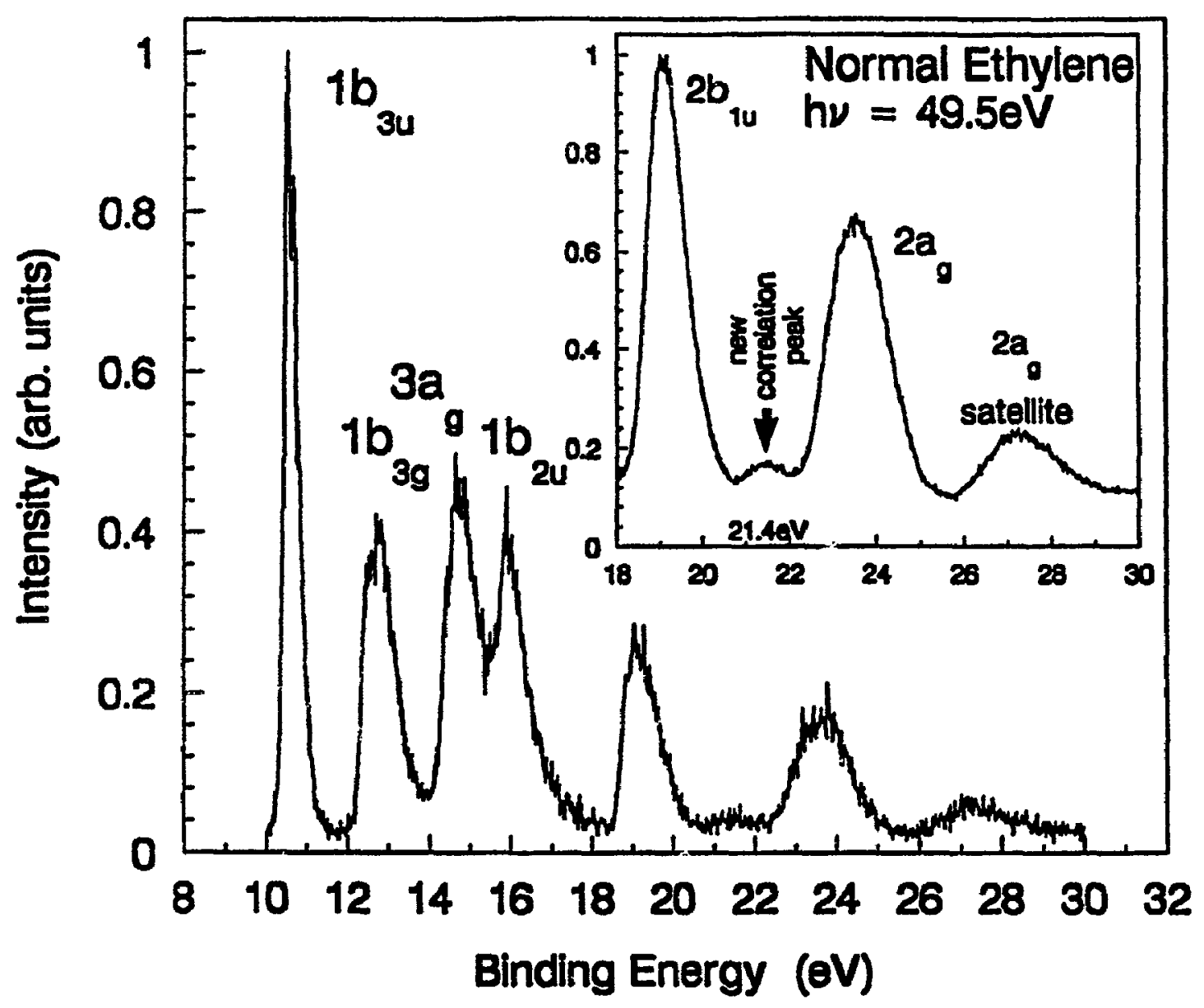

Figure 9: Main: A representative photoelectron spectrum of normal ethylene taken at $49.5 \mathrm{eV}$ photon energy with a total energy resolution of $360 \mathrm{meV}$ fwhm. Ins -rt: A section of the photoelectron spectrum of ethylene obtained with several scans highlighting the new correlation peak at $21.4 \mathrm{eV}$ binding energy between the $2 b_{1 u}$ and $2 a_{g}$ peaks. The peak at $27.4 \mathrm{eV}$ binding energy is produced by intrinsic correlations $[29,31]$. The spectra as shown ase not corrected for transmission effects. 


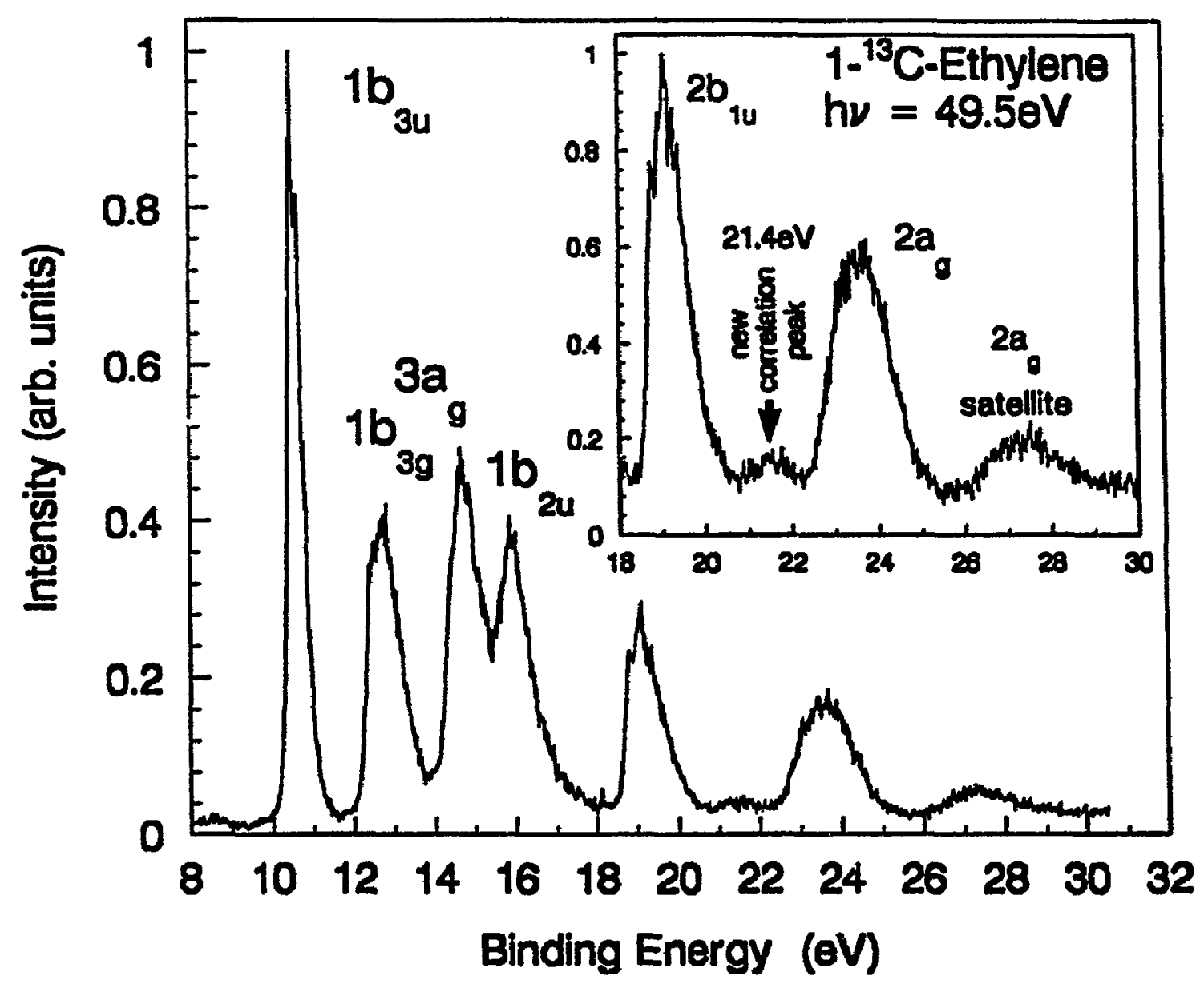

Figure 10: Main: A representative photoelectron spectrum of $1-{ }^{13} \mathrm{C}$-ethylene taken at $49.5 \mathrm{eV}$ to show that the SPES is similar to that of normal ethylene, having the same new correlation peak at the same energy with the same intensity. Insert: Another spectrum obtained with better statistics to highlight the new correlation peak. The spectra as shown are not corrected for transmission effects. 
(erderbaum ot al [61] (see Table i) and Figure 16) did predict a peak between tho $2 b_{1 u}^{-1}$ and $2 a_{y}^{-1}$ main praks, associated with the $2 b_{1 u}^{-1}$ peak. The new correlation peak has a well-defined peak shape $\left(1.5 \in V^{r} f u h m\right)$ and is found to have significant intensity over a certain photon energy range. It should be noted that the same satellite peak is also observed in the synchrotron PES of ${ }^{13} \mathrm{C}$-labelled ethylene in the same range of photon energies (see Figure 10). An investigation of the pressure dependence of the photoionization cross-section has also shown that the correlation peak at $21.4 \mathrm{eV}$ is not a result of inelastic processes. The intensity ratio of the new correlation peak to the $2 b_{1 u}^{-1}$ main peak was found to vary by less than $4 \%$ over an order of magnitude of change in pressure. Nor is the new satellite caused by Auger processes or second order radiations, as the peak position (ie binding energy) was found to be constant and the majorit of the experiments were conducted at photon energies where second order radiation is inconsequential. And so, these studies clearly indicate that the new correlation peak at $21.4 \mathrm{eV}$ is not a result of any experimental artifacts.

The ratio of the new correlation peak intensity to the intensity of the $2 b_{1 u}^{-1}$ peak for normal ethylene as a function of photon energy is shown in Table 3 and Figure 11. The ratio of the new correlation peak to $2 b_{1 u}^{-1}$ for labelled ethylene is shown in Table 4 and Figure 12. The $2 b_{1 u}^{-1}$ main peak is tentatively chosen as the parent peak of the new correlation peak for convenience. The present results are independent of this choice since the photon energy dependences of the $2 a_{g} / 2 b_{1 u}$ 
Table 3: Transmission corrected satcllite/main $2 b_{14}^{-1}$ intensity ratios for the new correlation state at $21.4 \mathrm{cl}$ binding energy of normal et hylene.

\begin{tabular}{|c|c|c|c|c|c|}
\hline $\begin{array}{c}\text { Photon } \\
\text { Energy } \\
\text { (eV) }\end{array}$ & $\begin{array}{c}\text { Intensity } \\
\text { Ratioa } \\
( \pm \sigma)\end{array}$ & $\begin{array}{c}\text { Plioton } \\
\text { Energy } \\
\text { (eV) }\end{array}$ & $\begin{array}{c}\text { Intensity } \\
\text { Ratio } \\
( \pm \sigma)\end{array}$ & $\begin{array}{c}\text { Photon } \\
\text { Energy } \\
(\mathrm{eV})\end{array}$ & $\begin{array}{c}\text { Intensity } \\
\text { Ratio" } \\
( \pm \sigma)\end{array}$ \\
\hline $29.8^{T}$ & $0.192(0.047)$ & $56.7^{T}$ & $0.115(0.013)$ & $1: 31.1$ & $0.016(0.009)$ \\
\hline $31.8^{T}$ & $0.152(0.014)$ & $58.7^{T}$ & $0.101(0.021)$ & 121.3 & $0.028(0.013)$ \\
\hline $33.8^{T}$ & $0.106(0.020)$ & 59.5 & $0.108(0.007)$ & 126.4 & $0.021(0.015)$ \\
\hline $35.8^{T}$ & $0.104(0.024)$ & $60.7^{T}$ & $0.093(0.013)$ & 131.5 & $0.0333(0.021)$ \\
\hline $36.7^{T}$ & $0.092(0.021)$ & $62.7^{T}$ & $0.092(0.014)$ & 136.5 & $0.03: 3(0.013)$ \\
\hline $38.7^{T}$ & $0.114(0.017)$ & 64.5 & $0.109(0.009)$ & 141.6 & $0.025(0.011)$ \\
\hline 39.5 & $0.163(0.012)$ & $64.7^{T}$ & $0.074(0.014)$ & 146.8 & $0.024(0.010)$ \\
\hline $39.7^{T}$ & $0.156(0.015)$ & $60.7^{T}$ & $0.068(0.012)$ & 151.1 & $0.032(0.024)$ \\
\hline $40.7^{T}$ & $0.180(0.025)$ & $68.7^{T}$ & $0.076(0.017)$ & 151.9 & $0.031)(0.010)$ \\
\hline $41.8^{T}$ & $0.140(0.026)$ & 69.5 & $0.093(0.009)$ & 157.0 & $0.03 ;(0.018)$ \\
\hline $42.7^{T}$ & $0.140(0.030)$ & 70.6 & $0.071(0.025)$ & 162.1 & $0.033(0.017)$ \\
\hline $43.8^{T}$ & $0.145(0.019)$ & $70.7^{T}$ & $0.060(0.01 .3)$ & 167.1 & $0.035(0.015)$ \\
\hline 44.5 & $0.119(0.007)$ & 71.1 & $0.080(0.040)$ & $17: 2.2$ & $0.032(0.017)$ \\
\hline $44.7^{T}$ & $0.138(0.021)$ & $72.7^{T}$ & $0.074(0.016)$ & 177.2 & $0.029(0.015)$ \\
\hline $46.7^{T}$ & $0.106(0.021)$ & 75.6 & $0.041(0.020)$ & 182.4 & $0.028(0.016)$ \\
\hline $46.8^{T}$ & $0.105(0.017)$ & 80.7 & $0.043(0.014)$ & 187.6 & $0.030(0.016)$ \\
\hline $48.7^{T}$ & $\therefore .112(0.017)$ & 85.8 & $0.056(0.017)$ & 192.6 & $0.028(0.014)$ \\
\hline $48.8^{T}$ & $0.094(0.011)$ & 90.8 & $0.054(0.017)$ & 197.7 & $0.029(0.016)$ \\
\hline 49.5 & $0.102(0.007)$ & 91.1 & $0.025(0.018)$ & 202.7 & $0.028(0.020)$ \\
\hline $50.7^{T}$ & $0.088(0.017)$ & 95.9 & $0.055(0.014)$ & 207.8 & $0.035(0.019)$ \\
\hline $50.8^{T}$ & $0.092(0.014)$ & 101.1 & $0.058(0.013)$ & 212.9 & $0.010(0.010)$ \\
\hline $52.7^{T}$ & $0.096(0.014)$ & 106.1 & $0.047(0.012)$ & 217.8 & $0.010(0.010)$ \\
\hline $52.8^{T}$ & $0.124(0.018)$ & 111.2 & $0.060(0.011)$ & 222.9 & $0.022(0.017$; \\
\hline 54.5 & $0.108(0.007)$ & 116.3 & $0.030(0.016)$ & $40.8^{H}$ & $0.123(0.040)$ \\
\hline $54.7^{T}$ & $0.111(0.016)$ & & & & \\
\hline
\end{tabular}

a Quoted error limits are statistical errors from gaussian curve fitting alone and do not include systematic errors. Systematic errors due to the assumed baseline and transmission function are estimated to be less than $5 \%$ for the results obtained with the grasshopper and on the order of $10 \%$ for those obtained with the $3 m$-TGM.

$T$ Results from the $3 m$-TGM, all other values are from the grasshopper monochromator.

${ }^{H}$ He II result from reference [45]. 


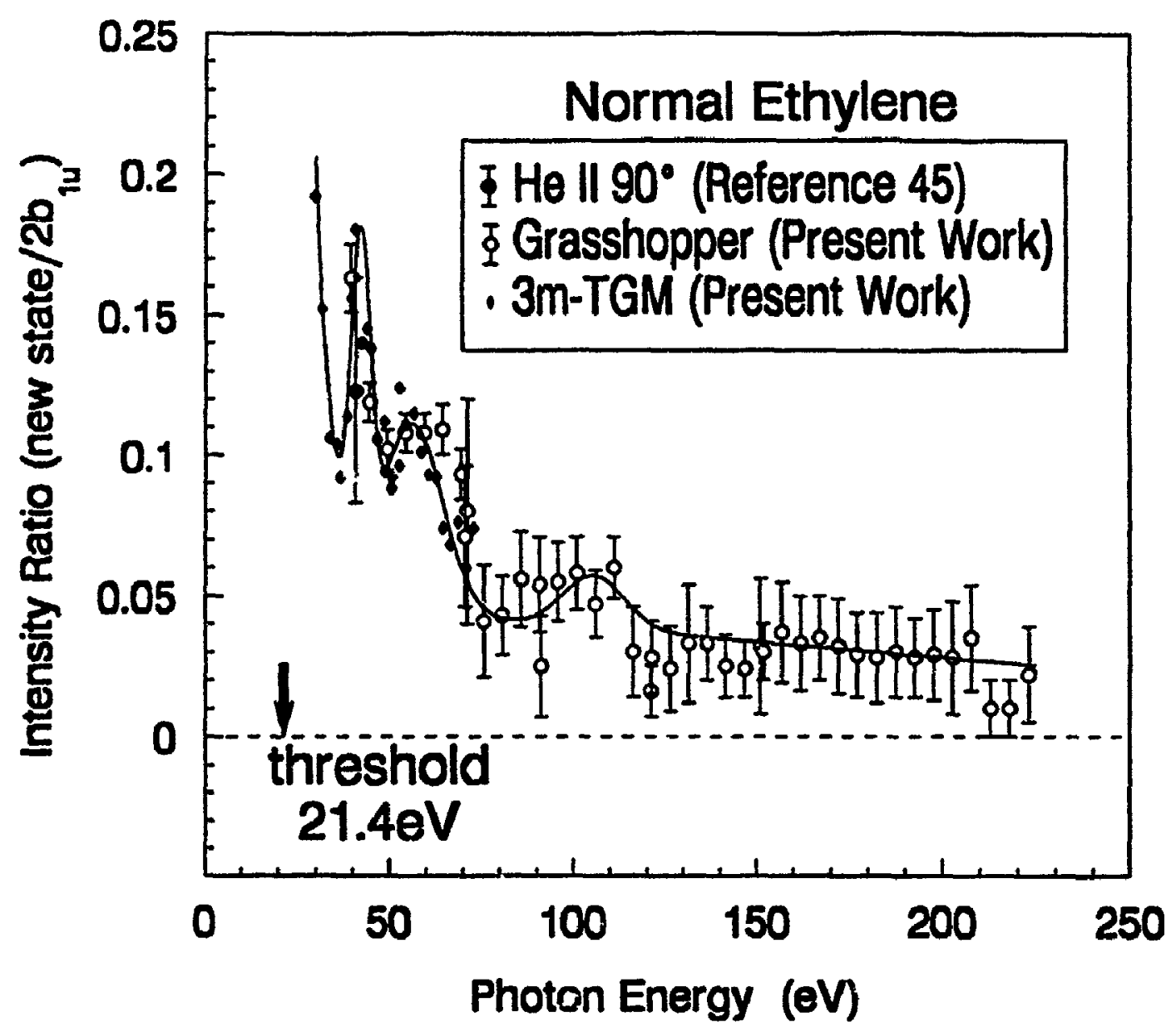

Figure 11: Ratio of the intensity of the new correlation peak to the intensity of the $2 b_{\mathrm{iu}}^{-1}$ peak as a function of photon energy for normal ethylene. The statistical errors involved in the measurements are quoted in Table 3 . All reported ratios are corrected for transmission effects. The He II result (solid circle with error bar) was calculated from a spectrum originally published in reference 45 and was not corrected for $\beta$-factor and transmission effects. The open circles with error bars are results from the grasshopper monochromator, the solid diamonds from the $3 \mathrm{~m}$-TGM and the solid line serves to highlight the resonance-like features at 42,57 and $105 \mathrm{eV}$ photon energy. 
Table 4: Transmission corrected satellite intensity ratios for $1-^{13}$ ( $^{\circ}$ ethylene for the ne" correlation peak at $21.4 \mathrm{eV}$ binding energy.

\begin{tabular}{|c|c|}
\hline $\begin{array}{c}\text { Photon Energy } \\
(e V)\end{array}$ & $\begin{array}{c}\text { Intensity Ratio }{ }^{\alpha}( \pm \sigma) \\
\text { satellite/main } 2 b_{1 u}^{-1}\end{array}$ \\
\hline 44.5 & $0.116(0.017)$ \\
49.5 & $0.106(0.010)$ \\
& $0.123(0.032)$ \\
54.5 & $0.109(0.013)$ \\
$58.8^{\tau}$ & $0.096(0.021)$ \\
& $0.126(0.063)$ \\
& $0.103(0.025)$ \\
59.5 & $0.107(0.023)$ \\
& $0.094(0.011)$ \\
64.5 & $0.102(0.012)$ \\
69.5 & $0.093(0.011)$ \\
& $0.094(0.018)$ \\
91.1 & $0.040(0.027)$ \\
121.7 & $0.028(0.028)$ \\
151.9 & $0.020(0.020)$ \\
\hline
\end{tabular}

${ }^{a}$ Quoted error limits are statistical errors from gaussian curve fitting alone and do not include systematic errors. Systematic errors due to the assumed baseline and transmission function are estimated to be less than $5 \%$ for the results obtained with the grasshopper and on the order of $10 \%$ for those obtained with the $3 m$-TGM.

$T$ The $58.8 \mathrm{eV}$ results are from the $3 \mathrm{~m}$-TGM, all other values are from the grasshopper monochromator. 


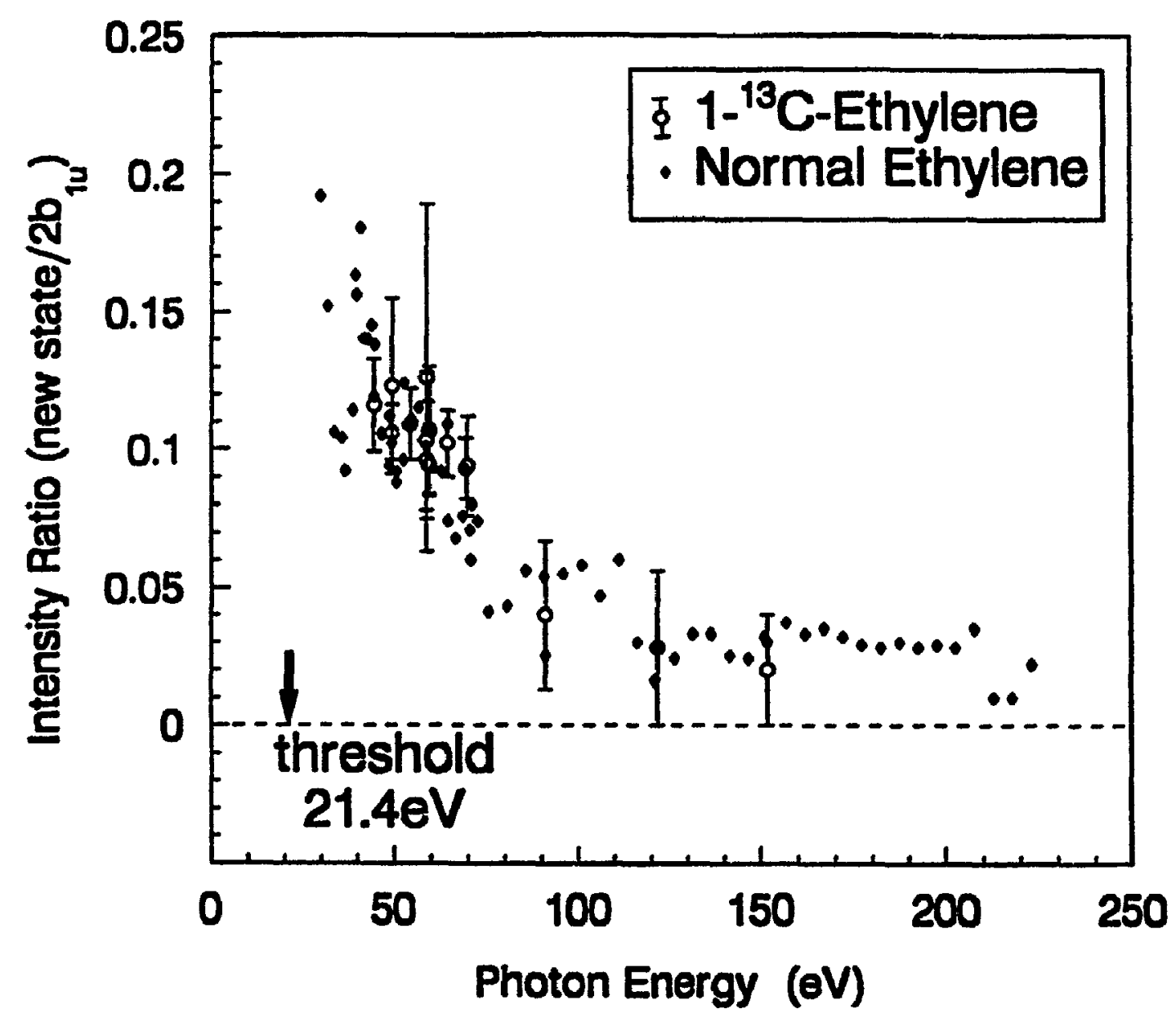

Figure 12: Intensity ratio of the new correlation peak to the $2 b_{1 u}^{-1}$ main peak as a function of photon energy for $1 .{ }^{13} \mathrm{C}$-ethylene. The ratio values (Table 4 ) have been corrected for transmission effects. The data are shown as open circles with error bars; the ratio values for normal ethylene are shown as solid diamonds. 
and $1 b_{2 u} / 2 b_{1}$, ratios are reosonably constant in the photon energy range of the current investigation. The experimentally derived mew cortedition peak/ $2 b_{11}^{-1}$ intensity ratio clearly shows a st rong phot on energy dependence and would apperar to be a result of "dynamic" correlations. The new peak has signiticant intensity only at lower photon energies, ic less than $70 \mathrm{cr}$. and is virtually unnoticrable beyond $150 \mathrm{eV}$, thus explaining why this peak was not observed in previous high resolution X-ray photoelectron spectra $[42,43,46]$ and the higher encrgy syuchrotron work [29]. Bieri and Asbrink detected this peak in their He II work [15], but did] not make any comment as to its origin. Their published experimental spertrum has been digitized and deconvoluted like the synchrotron PES to obtain a valus for the satellite to $2 b_{1 u}^{-1}$ main peak ratio which is also shown in Figure 11 . 'The large error bar associated with this point is indicative of the uncertainty resulting from the digitization of the published spectrum and the lack of knowledge of the. background and transmission characteristics of the spectrometer used. Also, the He II work was conducted at $\theta=90^{\circ}$, thus requiring an angular correction to the ratio. The differential cross sections for unpolarized He II radiation are of the form $[79]$ :

$$
\frac{d \sigma}{d \Omega}=\left(\sigma_{0} / 4 \pi\right)\left[1-(\beta / 4)\left(3 \cos ^{2} \theta-1\right)\right]
$$

where $\beta$ is the angular anisotropy parameter (otherwise known as the / $\beta$-paranteter), $\sigma_{0}$ is the partial ionization cross section and $\theta$ is the photoelectron ejection angle relative to the photon propagation direction. Since $\theta=90^{\circ}$, the differential cross 
section rerlucess to:

$$
\frac{d \sigma}{d \Omega}=\left(\sigma_{0} / 4 \pi\right)[1+(. t / 1)]
$$

and so the intensity ratio for the He II work is then of the form:

$$
\left[\frac{I(s a t)}{I\left(2 b_{1 u}\right)}\right]_{r} \|=\frac{\sigma_{0}(s a t)[1+(\beta(s a t) / 4)]}{\sigma_{0}\left(2 b_{1 u}\right)\left[1+\left(\beta\left(2 b_{1 u}\right) / 4\right)\right]}
$$

The differential cross section for polarized synchrotron radiation is [1,12]:

$$
\frac{d \sigma}{d \Omega}=\left(\sigma_{0} / 4 \pi\right)\left[1+(\beta / 2)\left(3 \cos ^{2} \theta-1\right)\right]
$$

where $\theta$ is the photoelectron ejection angle relative to the photon polarization axis. But the synchrotron work is conducted at the pseudomagic angle [4], and so requires no angular correction. The intensity ratio here is:

$$
\left[\frac{I(s a t)}{I\left(2 b_{1 u}\right)}\right]_{s y n c h r o t r o n}=\frac{\sigma_{0}(s a t)}{\sigma_{0}\left(2 b_{1 u}\right)}
$$

And so, the ratio of these two intensity ratio values is:

$$
\frac{\left[I(s a t) / I\left(2 b_{1 u}\right)\right]_{H e} I I}{\left[I(s a t) / I\left(2 b_{1 u}\right)\right]_{\text {synchrotron }}}=\frac{[1+(\beta(s a t) / 4)]}{\left[1+\left(\beta\left(2 b_{1 u}\right) / 4\right)\right]}
$$

But the two results are seen to approximately agree within the experimental and calculation uncertainty (ie this double ratio is approximately 1), and hence there is some evidence that $\beta($ sat $) \approx \beta\left(2 b_{1 u}\right)$, supporting the suggestion that the $2 b_{1 u}^{-1}$ peak could be the parent peak of the $21.4 \mathrm{eV}$ correlation peak.

There are several interesting trends to note regarding the photon energy dependence of the intensity of this new correlation peak of ethylene at $21.4 \mathrm{eV}$ 
(see Table 3 and Figure 11 ): (a) the general trend is increasing satcllite/:2b-11 intensity ratio as the threshold $\left(21.4 a^{\circ}\right)$ is approached: (b) superimposerl on th.s general trend are three "resonance-type" features located at photon conergies of $42 \mathrm{eV}, 57 \in V$ and $105 \mathrm{Cl}^{\circ}$ (corresponding to wavelengths of 2995. 2Is and $118 \AA$, respectively): the ejected elect rons then have wavelengt hs of $2.70,2.06$ and $1.34 \AA$, respectively; and (c) there is a consistent agreement between the two sets of experimental data obtained on two different beamlines; and, in addition, the only previous report of this correlation peak using Ifr II radiation shows agrerement. with the synchrotion results, indicating that the results obtained in the present study are not caused by any experimental artifact.

Following the general trend (a), this new correlation peak at $21.4 \mathrm{el}^{\prime}$ would appear to be produced by dynamic correlations. The exact, type of dynamic correlation mechanism is not apparent. Increasing satellite intensity towards threshold $(21.4 \mathrm{eV})$ is usually associated with continuum state configuration interaction [5], otherwise known as conjugate shake-up [79]. In shake-up theory [7], the outgoing photoelectron "excites" an outer valence electron to a higher discrete state; whereas, in conjugate shake-up [79], the outgoing photoelectron "excites" the" outer valence electron into a continuum state, leaving itself "trapped" in a higher discrete state. This behaviour can be qualitatively explained by considering the overlap between bound and continuum states which increases as the threstiolit. approached. However, in the atomic systems where conjugate shake-up has been 
olserved (og Ho [17.s0.si]. li [11] and . Ve [12]). the photon energy dependence of the satellite intensity is smoothly increasing to within approximately $10 \mathrm{eV}$ of threshold. A smooth trend is not the case for the new correlation peak at $21.4 \mathrm{Cl}$ since broad "resonance-type" features are also observed in the photon energy depondence curve (b). It is possible that these structures result from shape resonance [82] o: interchanncl coupling $[5,8,9,34]$; however, the only well-documented main features (as far as is known) of interchannel coupling in atomic systems [8] have shown widths in their resonance peaks of approximately $0.2-0.5 \mathrm{eV}$ fwhm, which is much siualler than the widths (approximately $8 \mathrm{eV} f w h m$ ) observed in ethylene (see Figure 11). Interchannel coupling refers to the autoionization of doubly excited states into different satellite channels $[8,18]$. Thus, it may be argued that the large widths of the resonance features in ethylene simply reflect the higher density of doubly excited states in a molecular system as compared to an atomic system.

It is possible that these "resonance-type" features result from one or a combination of the following processes: (1) shape-resonance [83,84]; (2) interchannel coupling $[8,9,34]$; or $(3)$ "orbital motion" of the departing photoelectron.

Shape-resonances have 'ween observed routinely in the ionization of outer valence orbitals which are generally well-characterized by single hole ion states. Shape resonance is so-called because it is the shape of the molecular potential energy curve that causes the phenomenon [83]. The electron is "trapped" in the 
potential well and a quasistationary wase is produced from the constructive interferences of waves reflected back and forth in the potential well [i3]. Since shape-resonance theory, or the continum multiple scattering method [8.4]. is effectively a single-particle theory, it cannot be applied rigorously to the photon energy dependence of correlation (satellite) states which require a many-body description. However, shape-resonance has been used as a rationalization of broad structures in the inner valence satellite region of $N_{2}$ [85]. If it is assumed that shape-resonance is a valid qualitative explanantion for the large widths $(8 \mathrm{cV}$ $f w h m)$ of the resonance-like features in ethylene, it is still quit" cifficult to explain the multiple resonance features in a single ionization channel.

Interchannel coupling, or autoionization from doubly excited neutral states, appears to be a more feasible explanation. The high density of doubly excited states may account for the broad resonance features observed in the photon energy dependence of the new correlation peak to $2 b_{1 u}^{-1}$ intensity ratio. However, it is not clear why the density of doubly excited states in ethylene would be concentrated in certain enragy regions (ie 42, 57 and $105 \mathrm{eV}$ ). The total photoabsorption data of Lee et al [86] shows a smooth trend over $180-650 \AA(69-19 \mathrm{eV})$ and no sharp structures are observed. As far as is known, there is only one published experimental report [87] on the inner valence partial photoionization cross sections of ethylene. Data obtamed by Brennan et al [87] for the $2 a_{y}^{-1}$ and $2 b_{1 u}^{-1}$ partial photoionization cross sections, within experimental error, do not show any clear 
structures in the $25-100$ e photon energy range. Partial photoionization cross sections for ionization out of the $1 b_{2 t}$ orbital of ethylene have been reported by (irimm a 1 l [88]. but none for ionization from the $2 a_{g}$ inner valence orbital. In the photon energy range investigated by Grimm et al $(12-26 \in V)$, no structure is observed in the experimental partial cross sections nor in the continuum multiple scattering calculations. Thus, of the data available, there is no strong evidence to corroborate interchannel coupling. More experimental work in these areas is clearly necessary.

The seeming regularity of the resonance features in the photon energy dependence of the $21.4 \mathrm{eV}$ correlation peak can be viewed as oscillations superimposed on an otherwise smoothly increasing cross section towards threshold. These oscillations in the cross section are suggestive of some dynamic "orbital motion" of the escaping photoelectron, similar in spirit to that proposed by $\mathrm{Du}$ and Delos [89]. The regularity of the resonance features can be rationalized in a semiclassical wavepacket picture of the "ejected" electron, wherein this "ejected" electron is correlated with some large semiclassical orbit. It has been shown experimentally and theoretically [89] that the absorption spectrum of an atom placed in a magnetic field exhibits oscillations. These oscillations were theoretically attributed [89] to closed (ie periodic) classical orbits of the electron in the combined Coulomb and magnetic fields. The basis of these theoretical rationalizations is Gutzwiller's formula [89], which relates periodic classical orbits with oscillations 
in the density of states of a quant um system. It is therefore tempting to postulate. a similar rationalization for the oscillations observed in the present experiment (see Figure 11); however. an important distinction should be acknowledged. In the present experiment, there is no appreciable external magnetic field and thus it is not clear how a classical orbit for the outgoing electron can be supported. If, however, a classical orbit is in fact supported, then it must be caused by the peculiar electronic and nuclear configurations in the correlation state generated during the photoionization process. And so, the efforts to identify the symmetry of the $21.4 \mathrm{eV}$ correlation peak are clearly relevant (see Theoretical section).

As can be seen from Figure 13 , the new peak $/ 2 b_{1 u}^{-1}$ intensity ratio photon energy dependence can be approximated reasonably well with an exponential decay. Following the Becker-Shirley scheme [5], the intensity can then be decomposed into three components: an intrinsic correlation component (the constant value, 0.021 , which is similar to the behaviour of the major satellite at $27.4 \mathrm{eV}$ binding energy), a dynamic component (the exponential decay, like continuum state configuration interaction (CSCI) [5]) and an oscillating component. The intrinsic and dynamic components are shown in Figure 13 and the oscillating component in Figure 14. The constant (intrinsic) and the exponential (dynamic) were fitted to the data. The data can be seen to exhibit oscillatory behaviour around the exponential decay. The intensity ratio values ere defined as:

$$
\text { Intensity Ratio }=a\left(e^{-\epsilon / b}+\lambda(\varepsilon)+c\right)
$$




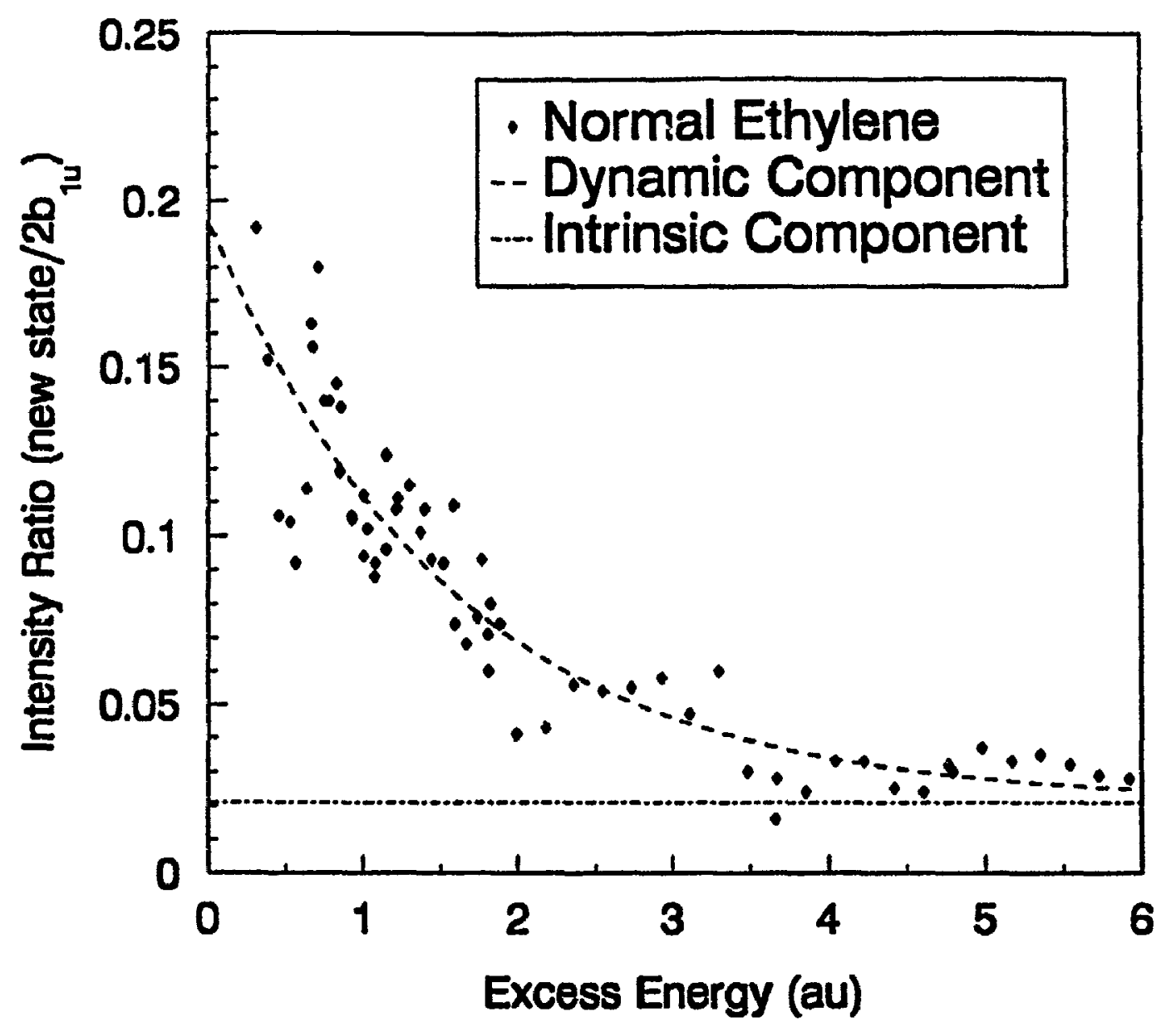

Figure 13: The decomposition of Figure 11. The intensity ratio values of the new correlation peak $/ 2 b_{1 u}^{-1}$ for normal ethylene are shown as solid diamonds. The photon energy dependence has been decomposed into three components: intrinsic, dynamic and oscillating (see text for details). The intrinsic component (constant) is shown as a dot-dash line; the dynamic component (exponential decay) is shown as a dashed line and the oscillating component is shown in Figure 14. The data are plotted versus scaled excess energy. The threshold energy of $21.4 \mathrm{eV}$ has been subtracted from the photon energies, with the results being converted to atomic units by dividing the differences by $27.21 \mathrm{eV}$. 
where $a, b$ and $c$ are constants (obtained from litting to the intensity ratio dat a) and $\Xi$ is the scaled excess energy (the threshold energy of 21.1 , 1 is subt racted from the photon energies. the resulting values are then converted to atomic units by dividing by $27.21, V)$. The components can then be seen to be: intrinsic = $a c$, dynamic $=a \epsilon^{-\varepsilon / b}$ and oscillating $=a \lambda(\varepsilon)$. The values of the constants are: $a=(0.173 \pm 0.010), b=(1.55 \pm 0.21)$ and $c=(0.121 \pm 0.031)$

The intrinsic and dynamic components were subtracted from the data. 'The resulting pure oscillating component was then converted from scaled excess conergy (au) to wavenumbers, $k\left(\AA^{-1}\right)$, and the function, $\lambda(k)$, is plotled in Figure 14 . The relationship between wavenumbers and kinetic energy is given by:

$$
k=\sqrt{2 m E_{k} / \hbar^{2}}
$$

The parameters from the curve fitting were then used to generate a smooth curve for $\lambda(k)$ (see Fig. 14) with equally-spaced intervals of $k$ through a simple FORTRAN program. The oscillating component curve was then fourier transformed using a Fast Fourier Transform (FFT) routine through GhAF'TOOL to extract characteristic distances from the oscillations. 'Ihe FFT of we $\lambda(k)$ function, $\Lambda(r)$, is shown in Figure 15. The major characteristic distances are then $(0.6 \pm 0.1)$ and $(2.2 \pm 0.2) \AA$, which are of molecular dimensions as the $C^{\prime}-C^{\prime}$ bond length in ethylene is $1.34 \AA[64]$. Errors on the distances are estimated from the drift of tho FFT peaks observed when $k \lambda(k)$ vs $k$ and $k^{3} \lambda(k)$ vs $k$ were fourier transformurd. 


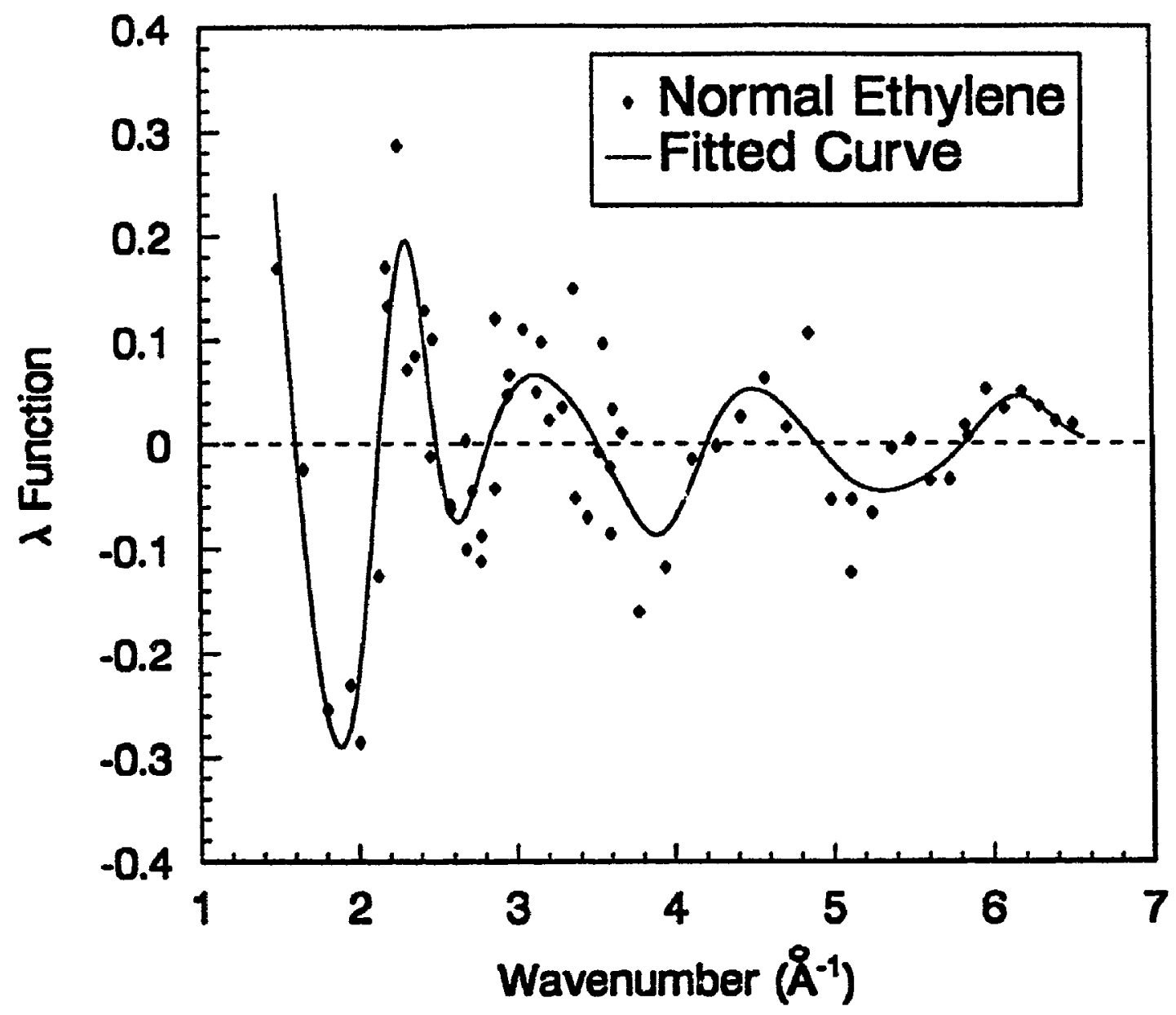

Figure 14: The oscillating part of the decomposition of Figure 11. The $\lambda(k)$ function (plotted in $\AA^{-1}$ ) is obtained by subtracting the constant and the exponential decay from the intensity ratio values and then converting to wavenumbers. The solid diamonds are the experimental values and the solid line is a fitted curve, generated from fitting parameters. 


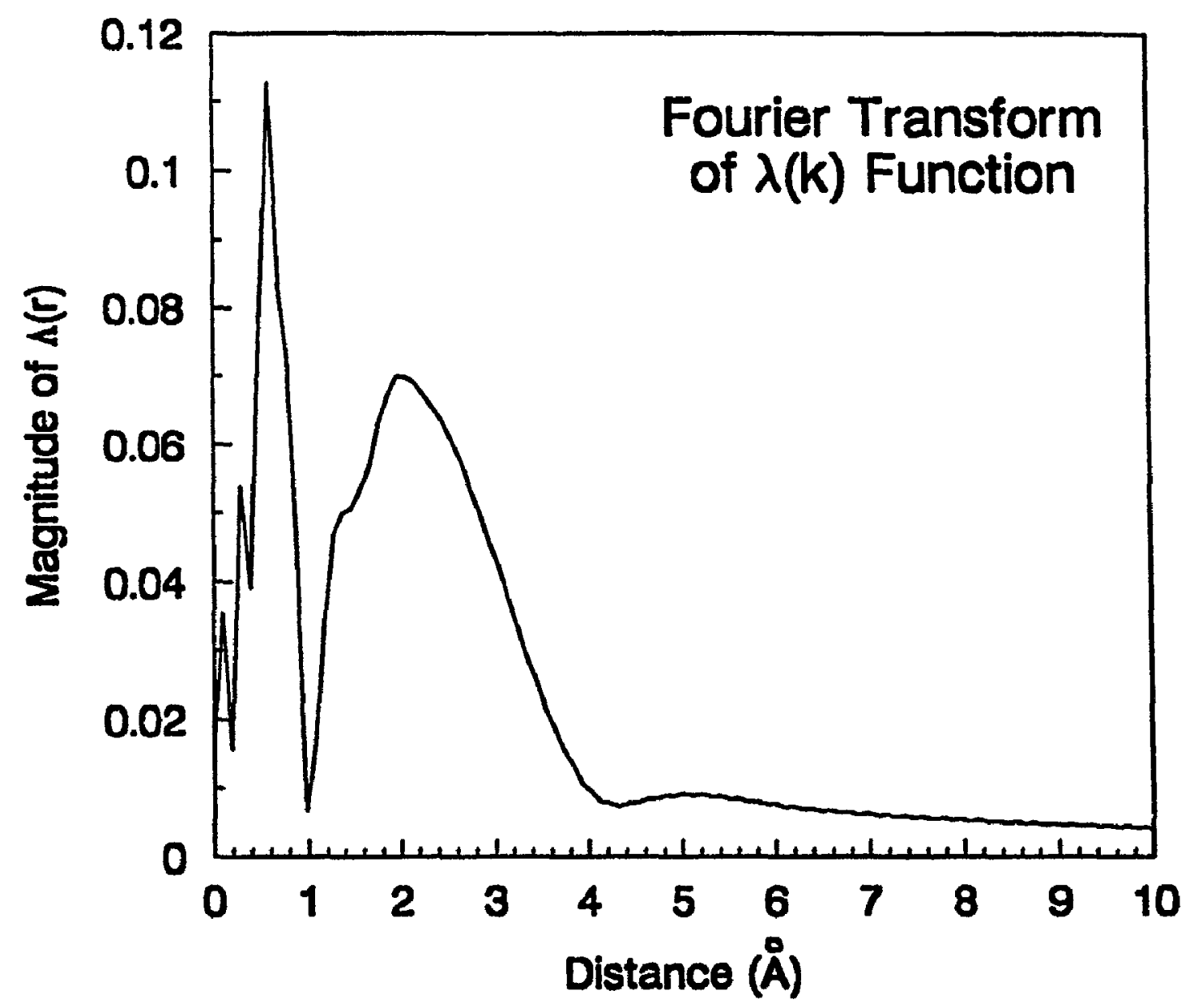

Figure 15: The $\Lambda(r)$ function is the Fast Fourier Transform (FFT) of $\lambda(k)$. Two major characteristic distances are seen for the oscillating component, with values of $(0.6 \pm 0.1)$ and $(2.2 \pm 0.2) \AA$. 


\section{Theoretical Calculations}

Recent theoretical calculations have become quite successful at predicting how the photoclectron spectrum of a molecular species should appear. The pole strength (ionization probability) for the $j$ th ionic state is defined by the square of the norm:

$$
S_{3}{ }^{2}=\left\|<\Psi_{\jmath}(N-1) \mid \Psi(N)>_{N-1}\right\|^{2}=\left(\left\|\sum_{p, q} C_{p j}^{*} D_{q} s_{p q}\right\|\right)^{2}
$$

where

$$
s_{p q}=<\Phi^{(p)}(N-1) \mid \Phi^{(q)}(N)>_{, N-1}
$$

and $\Psi_{3}(N-1)$ is the wavefunction of the $j$ th ionic state with $N-1$ electrons, $\Psi(N)$ is the ground state wavefunction of the neutral molecule with $N$ electrons, $C_{p j}$ is the coefficient that describes the extent of configuration mixing from the $p t h$ possible ionic configuration, $D_{q}$ is the CI coefficient for the $q$ th configuration of the neutral molecule, the subscript on the bracket indicates integration over only $N-1$ electrons, $\Phi^{(p)}(N-1)$ is the pth possible ionic configuration and $\Phi^{(q)}(N)$ is the qth possible configuration of the neutral molecule.

Any proper approximate wavefunctions for the cation and the neutral molecule can be used. If initial state configuration interaction (ISCI) and final ionic state configuration interaction (FISCl) are considered, correlation peaks arise through transitions to high-energy states of the same symmetry as the primary hole state. Within the dipole approximation, the intensity ratio of the $j$ th satellite (correla- 
tion) peak to the hth primary peak for a given symmetry is given by [90]:

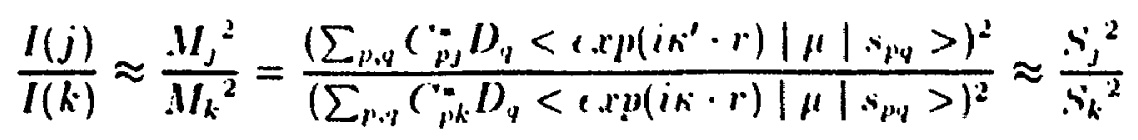

where the.$M$ s are the transition moments [31]. $\mu$ is the dipole operator and $e^{\text {in.r }}$ is the continuum wavefunction of the outgoing electron. This result assumes that the dipole matrix elements for the $j$ th satellite (correlation) peak and the hith prinary peak do not vary significantly. Futhermore, the coupling of channels in the continuum is neglected [90]. If only FISCI is considered, a single determinant wavefunction is used for the initial neutral state. For the $k$ th primary hole state, one of the coefficients for a $0 \mathrm{p}-1 \mathrm{~h}$ ( 0 particle - 1 hole) configuration is usually dominant. In a correlation state, all of those $0 \mathrm{p}-1 \mathrm{~h}$ coefficients are small, but usually one of the $0 \mathrm{p}-1 \mathrm{~h}$ coefficients is larger than the others. In this case, tho intensity ratio of the $j$ th satellite peak to the $k$ th primary peak involving the same $0 \mathrm{p}-1 \mathrm{~h}$ dominant configuration is $[59,90]$ :

$$
\frac{I(j)}{I(k)} \approx \frac{M_{j}^{2}}{M_{k}^{2}}=\frac{\left(\sum_{p} C_{p j}^{* *}<\exp \left(i \kappa^{\prime} \cdot r\right)|\mu| s_{p}>\right)^{2}}{\left(\sum_{p} C_{p k}^{*}<\exp (i \kappa \cdot r)|\mu| s_{p}>\right)^{2}} \approx \frac{C_{k j}^{2}}{C_{k k}^{2}}
$$

The assumption that spectral intensities can be roughly approximated by pole strengths is quite crude and can sometimes be misleading [62,91]. It has been observed that with FISCI and without ISCI, the intensities were satisfactory in the aggregate but very wrong in detail [92]; however, with both FISCI and ISCI, the agreement was much better.

In the case of ethylene, it was found that quantitative comparison could be made between theory and experiment [29]. It has also been shown that the: 
theorctical understanding of the major satellite at $27.4 \mathrm{el}$ binding energy is on a firm footing [29,31]. The underst anding of the new correlation peak is still lacking.

Table 5 and Figure If show the results of a non-diagonal Green's function ralculation for ethylene by Cederbaun ef al [61]. The non-diagonal matrix allows for processes involving more than one specific orbital. More correlating configurations were included in the calculation, but a smaller basis set was used than an carlier study [59]. It can be seen that the calculation fails in its prediction of $\cdot$ a region of the major satellite, as the predicted structure is much too broad. The calculation, however, does predict the existence of the new correlation state at $20.32 \mathrm{eV}$ binding energy and as belonging to the ${ }^{2} B_{1 u}$ symmetry manifold. UnCortunately, overall, the calculation is unsuccessful in predicting the quantitative details of the inner valence region of the spectrum [29-31].

Table 6 and Figure 17 show a recent Multi-Reference Singles and Doubles Configuration Interaction (MRSDCI) calculation by Murray and Davidson [60]. This calculation involved many more configurations, 433000 , than any other previous study, which generally used less than 500 . It can be seen that the calculation is quite successful at predicting the overall spectrum [29]. It can also be seen that the calculation correctly predicts the major satellite peak, including its intensity with respect to the $2 a_{g}^{-1}$ main peak (see Figure 17). In the binding energy region 27 to $29 \mathrm{eV}$, only ${ }^{2} A_{g}$ satellites are seen (see Table 6), further confirming earlier CI calculations [59]. in contrast to previous assignments $[63,64]$ that suggested 
that this correlation peak is of $2 B_{1 u}$ symmetry. For the new correlation peak. the calculation offers ${ }^{2} B_{1 u}\left(20.3,1^{\circ}\right),{ }^{2} A_{y}\left(22.1 \mathrm{Cl}^{\prime}\right)$ and ${ }^{2} B_{2 u}(2.2 .2,1)$ as possible contributions: none of these. however, seems convincing.

Davidson and his group have further refined their calculations [30.31]. 'Iabls. 7 and Figure 18 show the results for a very recent MRSDCI calculation bascel on average natural orbitals (ANO) [30.31]. The basis set used was 196-('C']'O (196) contracted gaussian type orbitals). Intensity is given by the pole strengths, $s_{j}^{2}$. The important configurations are also given. The notation convention is megative* powers indicate holes, positive powers are particles. So. $(x)^{-2}(y)^{\prime}$ would represent two holes in $x$ and one particle in $y$, or a $1 p$-2h configuration. Again, the overall prediction is quite successful and especially so in the region of the major satedlite. From Table 7 it can be seen that the latest extended basis set calculation (196CGTO) establishes once again that in the region 27 to $29 \mathrm{eV}$ only ${ }^{2} A_{g}$ satellites of significant intensity are detected. For the new correlation peak at $21.4 \mathrm{eV}$, the MRSDCI-ANO calculation offers $a^{2} B_{1 u}$ correlation state at $20.3 \mathrm{eV}$ binding energy and $\mathrm{a}^{2} B_{2 u}$ correlation state at $22.1 \mathrm{eV}$. The dominant configuration for the ${ }^{2} B_{1 u}$ correlation state is $\left(3 a_{g}\right)^{-1}\left(1 b_{3 u}\right)^{-1}\left(1 b_{2 g}\right)^{1}$. For the ${ }^{2} B_{2 u}$ correlation state, the dominant configuration is $\left(1 b_{3 g}\right)^{-1}\left(1 b_{3 u}\right)^{-1}\left(1 b_{2 g}\right)^{1}$. Both of these states are oneparticle two-hole (1p-2h) states. These states can be considered to be related to the primary ionization events where an electron has dropped from a higher encrgy orbital to fill the initial hole and a $\pi$ to $\pi^{*}$ transition has occurred. Because these 
pairs of transitions would repuire little energy: it is easy to see how these states rould interact with the corresponding $0 \mathrm{p}$ - th states. Both of these predictions are more convincing in terms of intensity. Both of the predicted correlation peaks have an intensity ratio of approximately $10 \%$ with respect to the predicted intensity of the $2 h_{1 u}^{-1}$ main peak. Unfortunately, narrowing down the possibilities between these two correlation states is not possible at this time $[30,31]$. 
Table 5: Non-diagonal Green's Function calculation for the PESS of ethylene. Pole strengths are the theoretically predicted relative intensities. From reference (il.

\begin{tabular}{|c|c|c|}
\hline Symmetry & $\begin{array}{c}\text { Ionization } \\
\text { Potenti.ul (eV) }\end{array}$ & $\begin{array}{c}\text { Pole } \\
\text { Strength }\end{array}$ \\
\hline$A_{g}$ & 13.79 & 0.897 \\
& 23.12 & 0.355 \\
& 23.83 & 0.273 \\
& 26.96 & 0.068 \\
& 28.05 & 0.079 \\
& 30.61 & 0.050 \\
& 30.88 & 0.012 \\
& 33.14 & 0.013 \\
\hline$B_{1 u}$ & 18.34 & 0.762 \\
& 20.32 & 0.029 \\
& 25.63 & 0.080 \\
\hline$B_{2 u}$ & 15.06 & 0.814 \\
& 17.83 & 0.015 \\
& 23.23 & 0.078 \\
\hline$B_{3 g}$ & 12.16 & 0.904 \\
& 26.56 & 0.023 \\
\hline$B_{3 u}$ & 9.76 & 0.917 \\
\hline
\end{tabular}




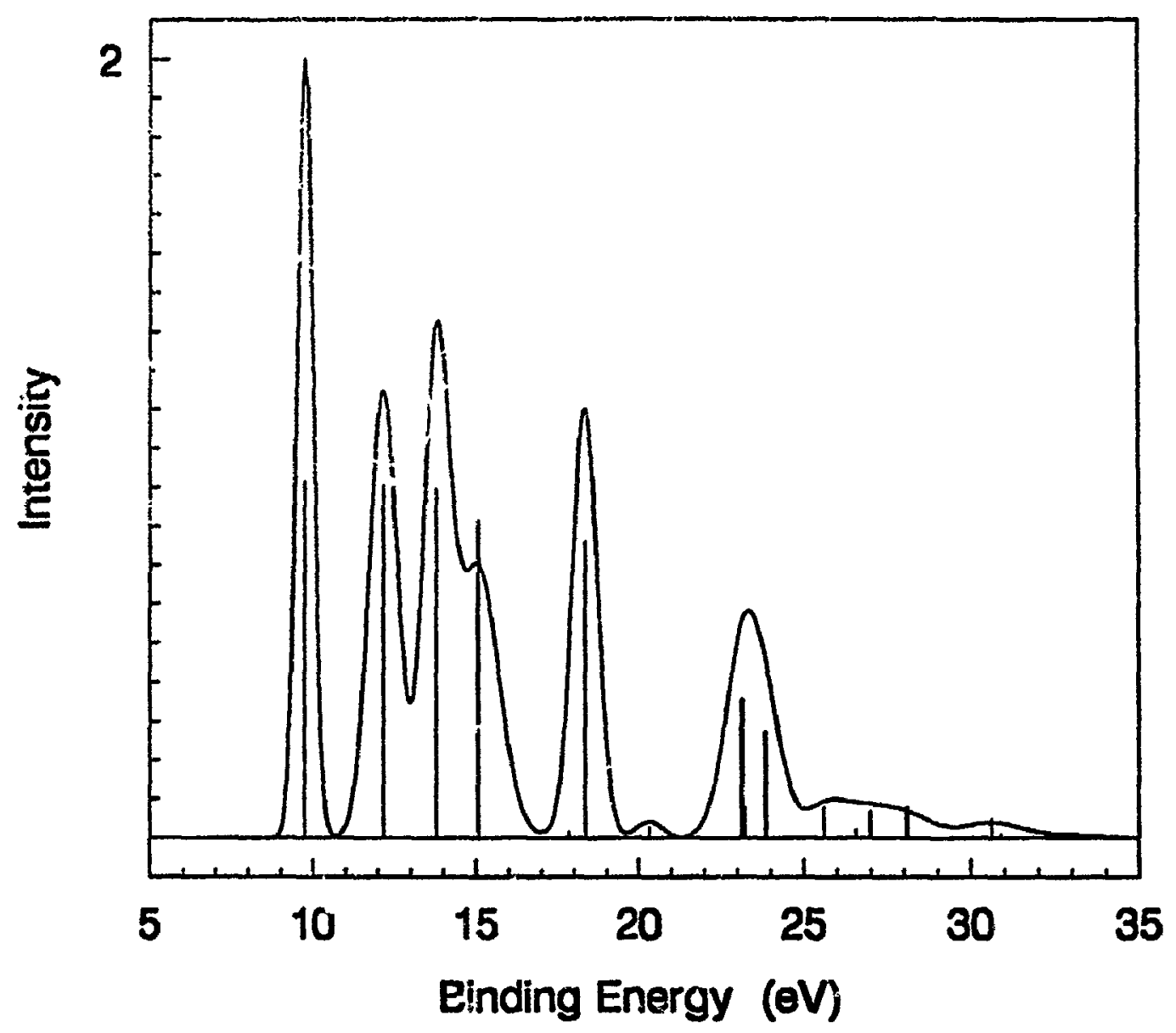

Figure 16: Non-diagonal Grten's Function caiculation of the PES of ethylene. The polez are show! as sticks; the overa!! curve was generated by ronvoluting the pole strengths with gaussian peaks whose widthe were consistent with the experimental values. From seference 61 . 
Table 6: The calculation by Murray and Davidson (the primary peak of eact. symmetry has been aligned with experiment). Root index refers to the number of predicted peaks within that symmetry - only those with an intensity greater than 0.01 are considered. The intensity is approximated by squaring the ('I expansion coefficients, $C_{k}[60]$.

\begin{tabular}{|c|c|c|c|}
\hline Symmetry & $\begin{array}{c}\text { Root } \\
\text { Index }\end{array}$ & $\begin{array}{c}\text { Energy } \\
(\mathrm{eV})\end{array}$ & $\begin{array}{c}\text { Intensity } \\
C_{k}^{2}\end{array}$ \\
\hline$A_{g}$ & 1 & 14.4 & 0.84 \\
& 2 & 22.1 & 0.02 \\
& 3 & 23.7 & 0.44 \\
& 4 & 24.6 & 0.04 \\
& 5 & 25.0 & 0.02 \\
& 8 & 26.8 & 0.03 \\
& 11 & 28.1 & 0.19 \\
& 13 & 28.9 & 0.01 \\
\hline$B_{1 u}$ & 1 & 19.2 & 0.66 \\
& 2 & 20.3 & 0.07 \\
& 4 & 24.7 & 0.09 \\
\hline$B_{2 u}$ & 1 & 15.9 & 0.77 \\
& 2 & 18.1 & 0.02 \\
& 3 & 22.2 & 0.05 \\
\hline$B_{3 g}$ & 1 & 12.9 & 0.85 \\
& 5 & 29.6 & 0.04 \\
\hline
\end{tabular}




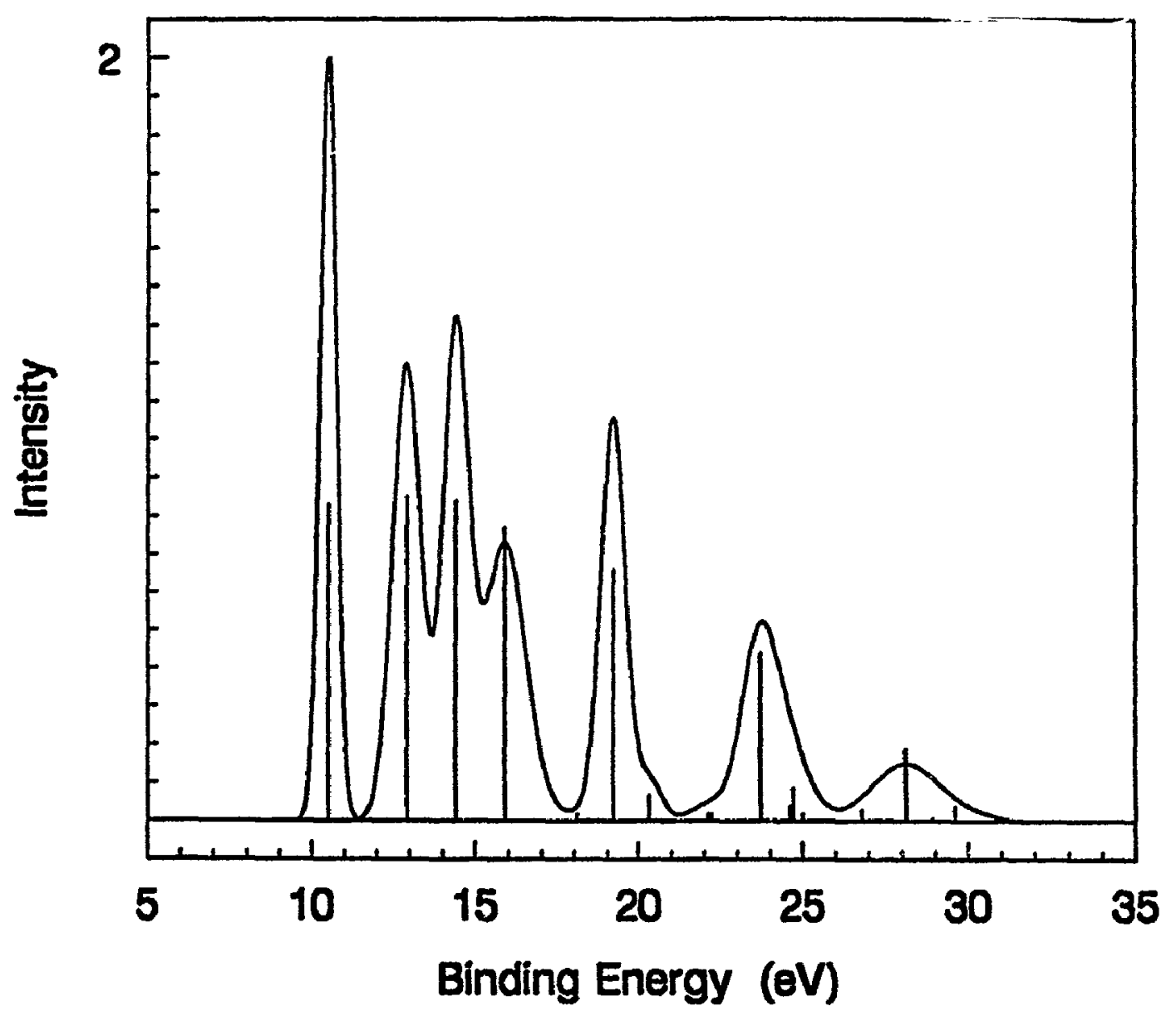

Figure 17: The calculation of Murray and Davidson from reference 60. 
Table 7 : The calculated line positions and intensities for the PLS of ethylene". (The MRSDCI-ANO calculation.) From reference 31.

\begin{tabular}{|c|c|c|c|c|}
\hline Symmetry & $\begin{array}{l}\text { Root } \\
\text { Index }\end{array}$ & $\begin{array}{c}\text { Ionization } \\
\text { Energy }^{b}\left(\mathrm{e}^{2} \mathrm{~V}\right)\end{array}$ & $\begin{array}{c}\text { Intensit } y^{\circ} \\
s y^{2}\end{array}$ & Important ('onfigurations \\
\hline$\overline{A_{g}}$ & 5 & $\begin{array}{l}14.1 \\
23.4 \\
24.6 \\
25.1 \\
27.1 \\
27.6 \\
27.8 \\
28.64 \\
28.61 \\
28.9\end{array}$ & $\begin{array}{l}0.81 \\
0.19 \\
0.28 \\
0.02 \\
0.02 \\
0.01 \\
0.03 \\
0.01 \\
0.09\end{array}$ & $\begin{array}{l}0.93\left(3 a_{g}\right)^{-1} \\
0.16\left(3 a_{g}\right)^{-1}\left(1 b_{3 u}\right)^{-2}\left(1 b_{2 g}\right)^{2} \\
0.77\left(1 b_{3 u}\right)^{-2}\left(n a_{g}\right)^{1} \\
0.46\left(2 a_{g}\right)^{-1} \\
0.28\left(2 b_{1 u}\right)^{-1}\left(1 b_{3 u}\right)^{-1}\left(1 b_{2 g}\right)^{1} \\
0.59\left(1 b_{3 u}\right)^{-2}\left(n a_{g}\right)^{1} \\
0.55\left(2 a_{g}\right)^{-1} \\
0.45\left(2 b_{1 u}\right)^{-1}\left(1 b_{3 u}\right)^{-1}\left(1 b_{2 g}\right)^{1} \\
0.92\left(1 b_{3 u}\right)^{-2}\left(n a_{g}\right)^{1} \\
0.19\left(2 b_{1 u}\right)^{-1}\left(1 b_{3 u}\right)^{-1}\left(1 b_{2 g}\right)^{1} \\
0.15\left(2 a_{g}\right)^{-1} \\
0.71\left(2 b_{1 u}\right)^{-1}\left(1 b_{3 u}\right)^{-1}\left(1 b_{2 g}\right)^{1} \\
0.40\left(3 a_{g}\right)^{-1}\left(1 b_{3 u}\right)^{-2}\left(1 b_{2 g}\right)^{2} \\
0.34\left(1 b_{3 u}\right)^{-2}\left(n a_{g}\right)^{1} \\
0.16\left(2 a_{g}\right)^{-1} \\
0.92\left(1 b_{3 u}\right)^{-2}\left(n a_{g}\right)^{1} \\
0.14\left(2 b_{1 u}\right)^{-1}\left(1 b_{3 u}\right)^{-1}\left(1 b_{2 g}\right)^{1} \\
0.11\left(2 a_{g}\right)^{-1} \\
0.84\left(3 a_{g}\right)^{-1}\left(1 b_{3 u}\right)^{-1}\left(n b_{3 u}\right)^{1} \\
0.27\left(3 a_{g}\right)^{-1}\left(1 b_{3 u}\right)^{-2}\left(1 b_{2 g}\right)^{2} \\
0.20\left(2 b_{1 u}\right)^{-1}\left(1 b_{3 u}\right)^{-1}\left(1 b_{2 g}\right)^{1} \\
0.18\left(2 a_{g}\right)^{-1} \\
0.49\left(2 b_{1 u}\right)^{-1}\left(1 b_{3 u}\right)^{-1}\left(1 b_{2 g}\right)^{1} \\
0.43\left(1 b_{3 g}\right)^{-2}\left(n a_{g}\right)^{1} \\
0.37\left(3 a_{g}\right)^{-1}\left(1 b_{3 u}\right)^{-2}\left(1 b_{2 g}\right)^{2} \\
0.29\left(2 a_{g}\right)^{-1} \\
0.79\left(3 a_{g}\right)^{-1}\left(1 b_{3 u}\right)^{-1}\left(n b_{3 u}\right)^{1} \\
0.47\left(1 b_{3 u}\right)^{-2}\left(n a_{g}\right)^{1} \\
0.10\left(3 a_{g}\right)^{-1}\left(1 b_{3 u}\right)^{-2}\left(1 b_{2 g}\right)^{2} \\
0.09\left(2 a_{g}\right)^{-1} \\
0.03\left(3 a_{g}\right)^{-1} \\
0.71\left(1 b_{3 g}\right)^{-2}\left(n a_{g}\right)^{1} \\
0.27\left(3 a_{g}\right)^{-1}\left(1 b_{3 u}\right)^{-2}\left(1 b_{2 g}\right)^{2} \\
0.25\left(2 b_{1 u}\right)^{-1}\left(1 b_{3 u}\right)^{-1}\left(1 b_{2 g}\right)^{1} \\
0.24\left(1 b_{2 u}\right)^{-2}\left(n a_{g}\right)^{1} \\
0.20\left(2 a_{g}\right)^{-1}\end{array}$ \\
\hline
\end{tabular}


Table 7 continued

\begin{tabular}{|c|c|c|c|c|}
\hline Symmetry & $\begin{array}{l}\text { Root } \\
\text { Index }\end{array}$ & $\begin{array}{c}\text { Ionization } \\
\text { Energy }^{b}(\mathrm{eV})\end{array}$ & $\begin{array}{c}\text { Intensity }{ }^{\epsilon} \\
S_{j}{ }^{2}\end{array}$ & Important Configurations $^{d}$ \\
\hline$\overline{A_{g}}$ & 13 & 29.1 & 0.02 & $\begin{array}{l}0.76\left(1 b_{3 u}\right)^{-2}\left(n a_{g}\right)^{1} \\
0.35\left(3 a_{g}\right)^{-1}\left(1 b_{3 u}\right)^{-1}\left(n b_{3 u}\right)^{1} \\
0.19\left(2 b_{1 u}\right)^{-1}\left(1 b_{3 u}\right)^{-1}\left(1 b_{2 g}\right)^{1} \\
0.16\left(2 a_{g}\right)^{-1} \\
0.78\left(1 b_{2 u}\right)^{-1}\left(1 b_{3 u}\right)^{-1}\left(1 b_{1 g}\right)^{1} \\
0.37\left(1 b_{3 g}\right)^{-1}\left(1 b_{3 u}\right)^{-2}\left(1 b_{1 g}\right)^{1}\left(1 b_{2 g}\right)^{1} \\
0.26\left(1 b_{3 u}\right)^{-2}\left(n a_{g}\right)^{1} \\
0.10\left(2 a_{g}\right)^{-1}\end{array}$ \\
\hline$\overline{B_{3 u}}$ & 1 & 10.5 & 0.81 & $0.95\left(1 b_{3 u}\right)^{-1}$ \\
\hline$\overline{B_{2 u}}$ & $\begin{array}{l}2 \\
3\end{array}$ & $\begin{array}{l}15.9 \\
18.2 \\
22.1\end{array}$ & $\begin{array}{l}0.02 \\
0.06\end{array}$ & $\begin{array}{l}0.88\left(1 b_{2 u}\right)^{-1} \\
0.33\left(1 b_{3 g}\right)^{-1}\left(1 b_{3 u}\right)^{-1}\left(1 b_{2 g}\right)^{1} \\
0.11\left(1 b_{2 u}\right)^{-1}\left(1 b_{3 u}\right)^{-2}\left(1 b_{2 g}\right)^{2} \\
0.93\left(1 b_{3 g}\right)^{-1}\left(1 b_{3 u}\right)^{-1}\left(1 b_{2 g}\right)^{1} \\
0.14\left(1 b_{2 u}\right)^{-1} \\
0.80\left(1 b_{3 g}\right)^{-1}\left(1 b_{3 u}\right)^{-1}\left(1 b_{2 g}\right)^{1} \\
0.28\left(1 b_{2 u}\right)^{-1}\left(1 b_{3 u}\right)^{-2}\left(1 b_{2 g}\right)^{2} \\
0.22\left(1 b_{2 u}\right)^{-1}\end{array}$ \\
\hline$\overline{B_{1 u}}$ & $\begin{array}{l}2 \\
3 \\
4\end{array}$ & $\begin{array}{r}19.2 \\
20.3 \\
23.4 \\
24.3\end{array}$ & $\begin{array}{r}0.61 \\
0.06 \\
0.0050 \\
0.08\end{array}$ & $\begin{array}{l}0.82\left(2 b_{1 u}\right)^{-1} \\
0.41\left(3 a_{g}\right)^{-1}\left(1 b_{3 u}\right)^{-1}\left(1 b_{2 g}\right)^{1} \\
0.13\left(2 a_{g}\right)^{-1}\left(1 b_{3 u}\right)^{-1}\left(1 b_{2 g}\right)^{1} \\
0.91\left(3 a_{g}\right)^{-1}\left(1 b_{3 u}\right)^{-1}\left(1 b_{2 g}\right)^{1} \\
0.26\left(2 b_{1 u}\right)^{-1} \\
0.94\left(1 b_{3 u}\right)^{-2}\left(n b_{1 u}\right)^{1} \\
0.07\left(2 b_{1 u}\right)^{-1} \\
0.83\left(3 a_{g}\right)^{-1}\left(1 b_{3 u}\right)^{-1}\left(1 b_{2 g}\right)^{1} \\
0.26\left(2 b_{1 u}\right)^{-1} \\
0.23\left(2 b_{1 u}\right)^{-1}\left(1 b_{3 u}\right)^{-2}\left(1 b_{2 g}\right)^{2}\end{array}$ \\
\hline
\end{tabular}


Table 7 continued

\begin{tabular}{|c|c|c|c|c|}
\hline Symmetry & $\begin{array}{l}\text { Root } \\
\text { Index }\end{array}$ & $\begin{array}{c}\text { lonization } \\
\text { Energy }^{b}(\mathrm{eV})\end{array}$ & $\begin{array}{c}\text { Intensityc } \\
S_{j}{ }^{2}\end{array}$ & Important Configurations $\mathbf{s}^{d}$ \\
\hline$\overline{B_{3 g}}$ & $\bar{I}$ & $\begin{array}{l}12.9 \\
24.2\end{array}$ & 0.79 & $\begin{array}{l}0.92\left(1 b_{3 g}\right)^{-1} \\
0.16\left(1 b_{2 u}\right)^{-1}\left(1 b_{3 u}\right)^{-1}\left(1 b_{3 g}\right)^{1} \\
0.14\left(1 b_{3 g}\right)^{-1}\left(1 b_{3 u}\right)^{-2}\left(1 b_{2 g}\right)^{2} \\
0.70\left(1 b_{2 u}\right)^{-1}\left(1 b_{3 u}\right)^{-1}\left(1 b_{2 g}\right)^{1} \\
0.57\left(1 b_{3 g}\right)^{-1}\left(1 b_{3 u}\right)^{-2}\left(1 b_{2 g}\right)^{2}\end{array}$ \\
\hline$B_{2 g}$ & 1 & $17.2^{e}$ & 0.02 & $\begin{array}{l}0.94\left(1 b_{3 u}\right)^{-2}\left(n b_{2 g}\right)^{1} \\
0.11\left(3 a_{g}\right)^{-1}\left(1 b_{3 u}\right)^{-1}\left(n b_{1 u}\right)^{1}\end{array}$ \\
\hline $\begin{array}{l}B_{1 g} \\
A_{u} \\
\end{array}$ & $\begin{array}{l}1 \\
1\end{array}$ & $\begin{array}{l}34.6^{f} \\
43.7^{f}\end{array}$ & $\begin{array}{l}0 \\
0\end{array}$ & \\
\hline
\end{tabular}

a For each symmetry, the MRSDCI wavefunctions were used for the cation and the neutral molecule and all calculations were based on the average natural orbitals except for the $B_{1 g}$ and $A_{u}$ symmetries.

b The first root energies have been adjusted to experimental data separately for each symmetry. The same constant shift was used for the rest of the roots in that symmetry.

- The values less than 0.005 were ignored.

d The absolute values for the $\mathrm{CI}$ coefficients were taken.

- The energy of the ground state of the neutral molucule was taken as the average value $(-78.40869342$ hartrees $)$ from the other 5 -root calculation results. The second root of this symmetry lies at $25.9 \mathrm{eV}$ with an intensity less than 0.005 . $f$ The energy of the ground state of the neutral molucule was taken as the average value $(-78.40869342$ hartrees $)$ from the other 5 -root calculation results. The energy of this symmetry was from a $1 \mathrm{p}-2 \mathrm{~h} \mathrm{CI}$ calculation result [31]. The pole strength is less than $10^{-4}$. 


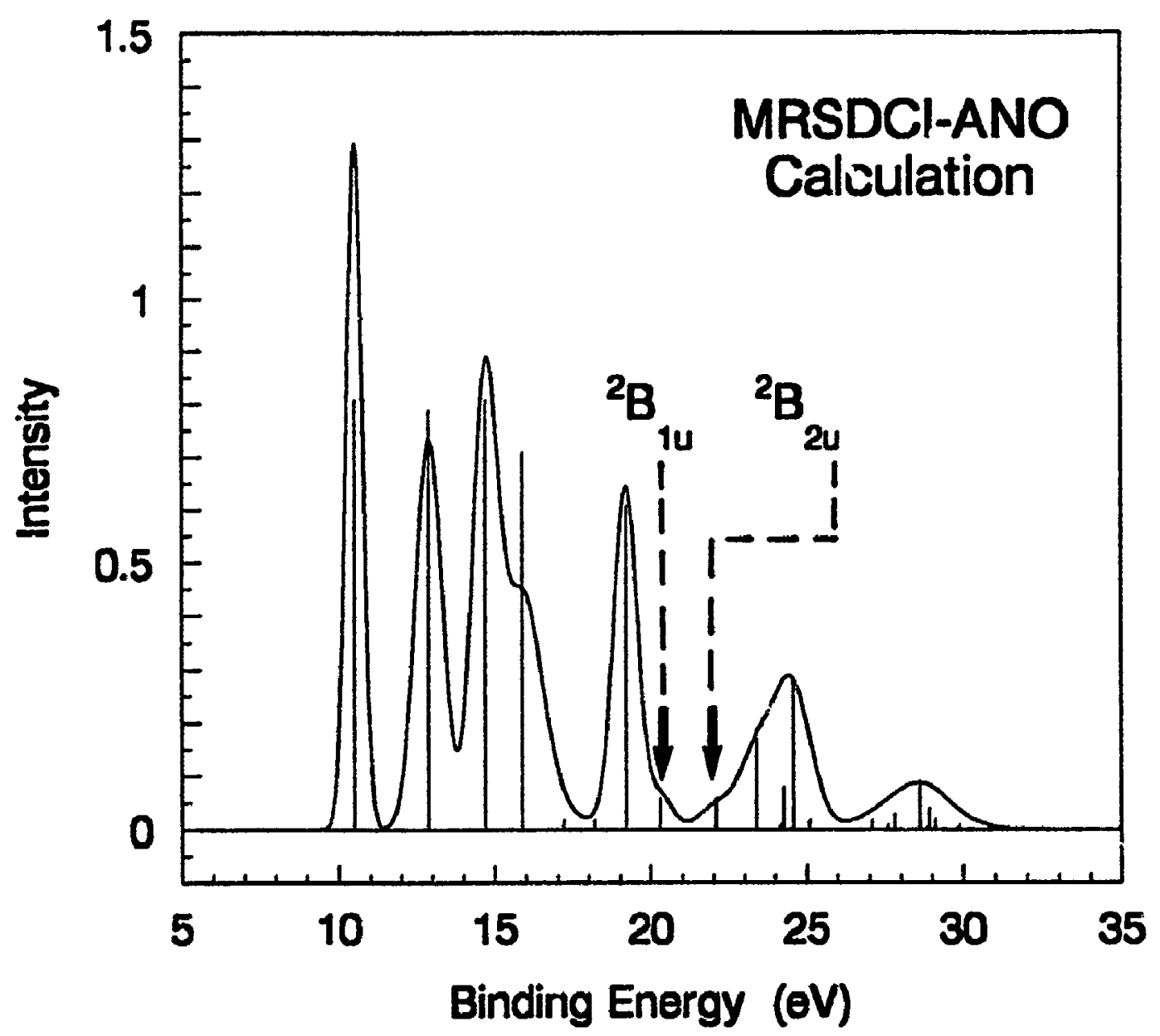

Figure 18: The MRSDCI-ANO calculation of Wang and Davidson from reference 31 . 


\section{Conclusions}

The Becker-Shirley schene [BS90] for the phenomenological classilication of correlation peaks has been shown to be feasible for the classitication of correlation peaks observed in synchrotron radiation photoelectron spectra [2!3-31].

The major correlation (satellite) peak of ethylene at $27 .+\mathrm{C} / \mathrm{binding}$ energy has been shown to be intrinsic in nature as its ratio with respect to its parent. peak has been shown to be effectively constant over a large photon 'nergy range. The symmetry assignment $\left({ }^{2} A_{y}\right)$ and the understanding of this feature is on firm theoretical ground.

The new correlation peak at $21.4 \mathrm{eV}$ binding energy has been shown to be dynamic. The features seen in the intensity ratio with respect to the $2 b_{11}^{-1}$ main peak are unexpected and still not fully understood. Whether this peak belongs to the ${ }^{2} B_{1 u}$ or ${ }^{2} B_{2 u}$ symmetry manifolds has yet to be determined. Also, the production mechanism for this peak is unclear as a combination of mechanisnts would be indicated from the shape of the intensity ratio curve (Figure 11).

Synchrotron photoelectron spectra of propene, 1-butene and 1,3-butadiene: have also been taken to study any chemical trends (as methyl groups are added) in satellite behaviour; though the analysis is presently incomplete. Maria SabayeMoghaddam is currently working on the analysis of acetylene spectra to investigate the correlation peaks that occur when a triple bond (ie two $\pi$ bonds) is present. A detailed systematic study of chemical effects in the synchrotron radiation photo. 
electron spectra of conjugated molecular systens will provide useful information needed to further the understanding of complex electron-e!.ctron correlations in atoms and molerules. 


\section{Appendix A: Second Order Radiation}

Table 8 and Figure 19 present transmission corrected intensity ratios of $\mathrm{Ne} \cdot 2 p$ for the second order radiation study. It can be seen that the second order intensity drops off quickly, to become inconsequential by $45 \mathrm{cV}$ photon energy. 
Table 8: Transmission corrected Intensity Ratio of second to first order radiations for the grasshopper monuchromator, derived from the $\mathrm{Ne} 2 p$ peak (ionization potential of $21.6 \mathrm{eV})$.

\begin{tabular}{|c|c|}
\hline Photon Euergy (eV) & Intensity Ratio \\
\hline 29.5 & $0.342 \pm 0.015$ \\
34.5 & $0.391 \pm 0.036$ \\
39.5 & $0.096 \pm 0.007$ \\
44.5 & $0.014 \pm 0.004$ \\
49.5 & $0.006 \pm 0.001$ \\
54.5 & $0.010 \pm 0.001$ \\
59.5 & $0.007 \pm 0.001$ \\
64.5 & $0.007 \pm 0.001$ \\
69.5 & $0.015 \pm 0.002$ \\
\hline
\end{tabular}




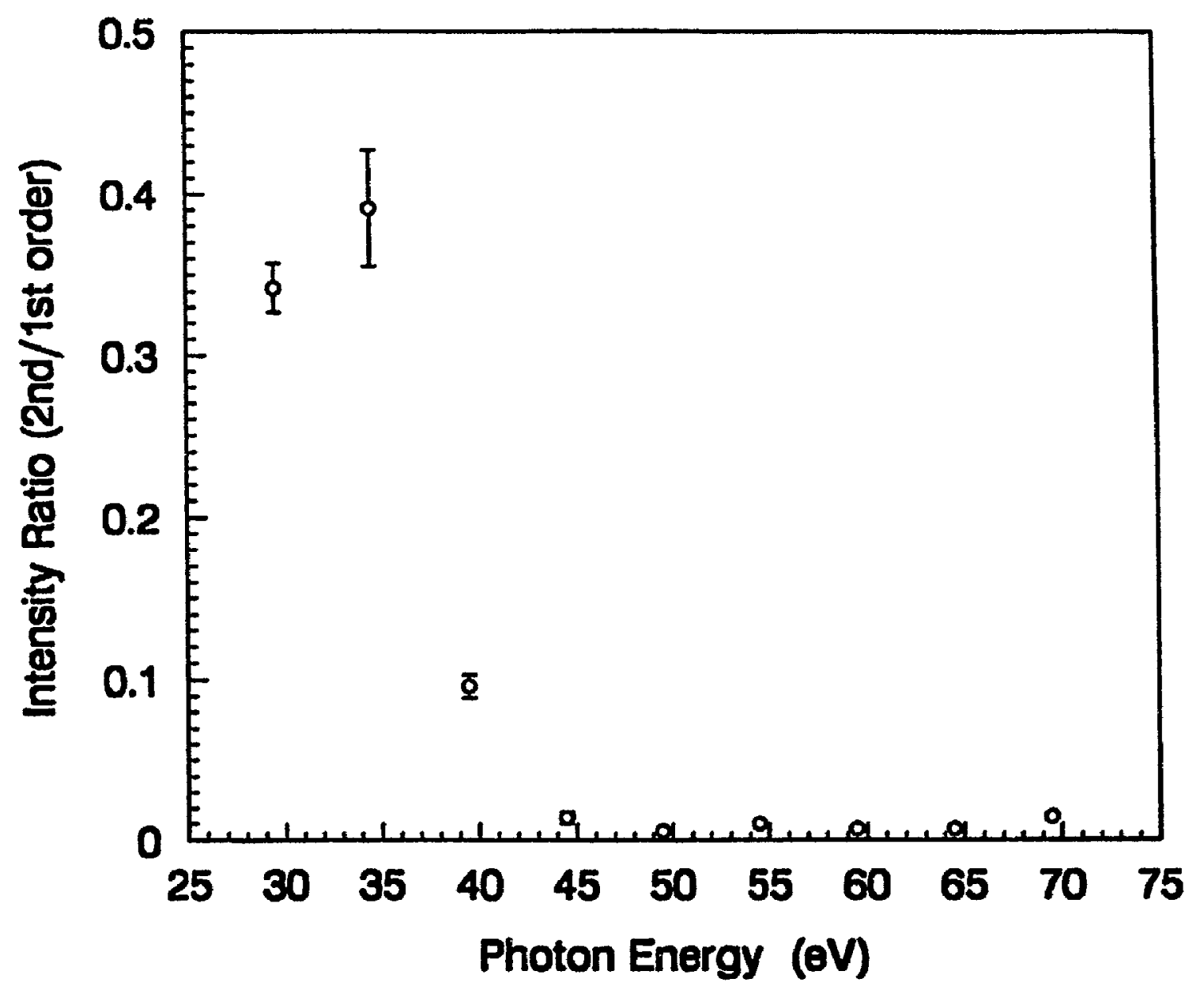

Figure 19: Transmission corrected Intensity Ratio of second to first order radiations for the grasshopper monochromator, derived from the Ne $2 p$ peak. 


\section{Appendix B: Ethylene Difference Spectrum}

Figure 20 shows the percent difference spectrum between Figures 5 and 6 in the binding conergy region of interest ( 18 to $30 \mathrm{eV}$ ). ic the inner valence. It is easily secoll that there is no truly discernible structure, and so, the two spectra are almost identical, except for effects from random processes, like noise. Thus, the substitution of ${ }^{13} \mathrm{C}$ ' for onc of the carbon atoms in ethylene causes no real difference in the photoelectron spectrum. 


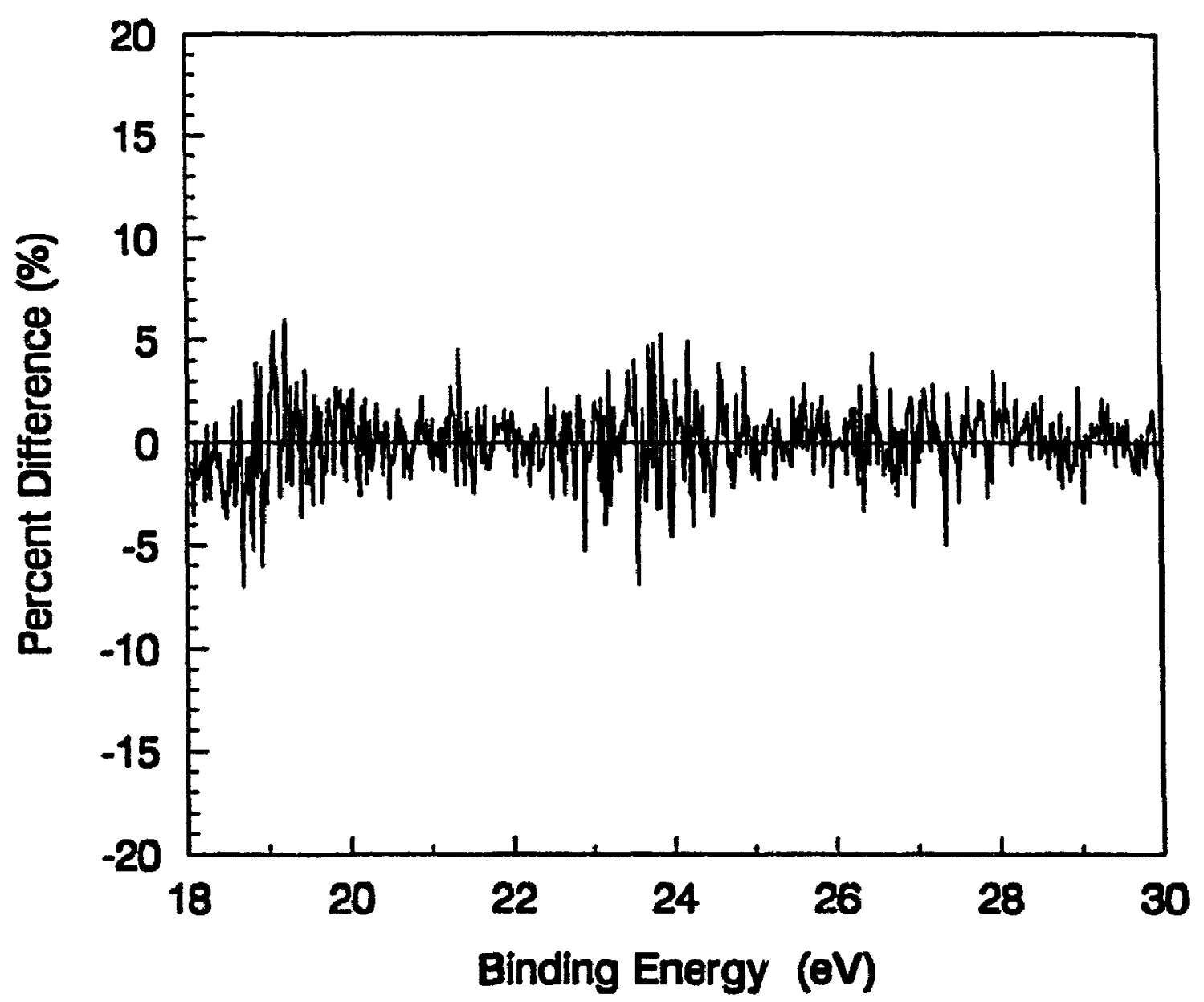

Figure 20: Percent Difference Spectrum (normal - labelled) derived from the SPES of normal and labelled ethylene taken at $59.5 \mathrm{eV}$ photon energy (see Fif':res 5 and 6). 


\section{References}

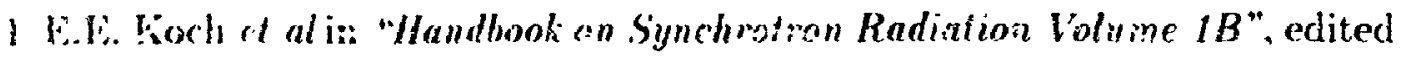
by L.K. Koch, North-Holland, Amst:erdam, 1983.

2 K.II. Tan, P.C. Cheng, G.M. Bancroft and J. W. McGowem, Can. J. Sper. $29134(1981)$.

3 G.iv. Bancroft, Can. Chem. Nows, p 15, june 1992.

4 J.D. Bozek, J.N. Cutler, C.M. Bancrofl. L.L. Cuatsworth. K.A. Tan, D.S. Yang and R.G. Cavell, Chem. Phys. Lett. 16.51 (1990).

5 II. Becker and D.A. Shirley, Phys. Scrip. T31 56 (1990).

6 F. Wuilleumier and M.O. Kralise, Phys. Rev. A 10242 (1974).

7 T.D. Thomas, Phys. Rex. Lett. 52417 (1984).

8 U. Becierer, R. Hölzel. H.G. Kerkhoff, B. Langer, D. Szostak and R. Wehlitz, Plyys. Res. Lett. $\underline{56} 11: 20$ (1936).

9 P.A. Heinann, U. Berker, H.G. Kerkhoff, B. Langer, D. Szostak, R.Wehlitz, D.W. Lindle, T A. Ferrett and D.A. Shiriey, Phys. Rev. A 343782 (1986).

10 A.D.O. Bawagan, B.J. Olsson, K.H. Tan, J.M. Cher and G.M. Bancroft, Chem. Phys. Lett. 139344 (299i). 
11 T.A. Ferrett. D.W. Lindle, P.A. Heimann. W.D. Brewer, U. Becker, H.C. Kerkhoff and D.A. Shirley, Physs. Rer. A $\cdots 3172(1987)$.

12 I. Becker et al (1989), quoted on page 1591 in V. Schmidt. Rep. Prog. Phys. 55 1483 (1992).

13 K.G. Dyall and F.P. Larkins, J. Phys. B $\underline{15} 203,219$ (1982).

14 U. Gelius, J. Eler, Spec. Relat. Phenom. 5985 (1974).

15 M.A. Brisk and A.D. Baker. J. Elec. Spec. Relat. Phenom. 7197 (1975).

16 M.Y. Adam, F. Wuilleumier, S. Krummacher, N. Sander, V. Schmidt and W. Mehlhorn, J. Elec. Spec. Relat. Phenom. 15211 (1979).

17 D.W. Lindle, T.A. Ferrett, U. Becker, P.H. Kobrin, C.M. Truesdale, H.G. Kerkhoff and D.A. Shirley, Phys. Rev. A 31714 (1985).

18 D.W. Lindle, P.A. Heimann, T.A. Ferrett, P.H. Liobrin, C.M. Truesdale, U. Becker, H.G. Kerkhoff and D.A. Shirley, Phys. Rev. A 33319 (1986).

19 U. Becker, H.G. Kerkhoff, D.W. Lindle, P.H. Kobrin, T.A. Ferrett, P.A. Heimann, C.M. Truesdale and D.A. Shirley, Phys. Rev. A 34 2858 (1986).

20 C.E. Brion, A.O. Bawagan and K.H. Tan, Chem. Phys. Lett. 13476 (1987).

21 H. Kossmann, B. Krässig, V. Schmidt and J.E. Hansen, Phys. Rev. Lett. $\underline{58} 1620(1987)$. 
22. [. Becker, B. Langer. H.G. Kerkhoff. M. Kupsch. D. Szostak, R. Wehlitz, P.A. Heimann, S.H. Lin, D.W. Lindle, T.A. Ferrett and D.A. Shirley, Phys. Rev. Lett. 60 $1490(1988)$.

23 (.E. Brion, A.O. Bawagan and K.H. Tan, Can. J. Chem. $\underline{66} 1877$ (1988).

24 J. Tulkki, Phys. Rev. Lett. $\underline{62} 281$ T (1989).

25 T.A. Forrett, D.W. Lindle, P.A. Heimann, H.G. Kerkhoff, U.E. Becker and D.A. Shirley, Phys. Rev. A $\underline{34} 1916$ (1986).

26 A. Reimer, J. Schirmer, J. Feldhaus, A.M. Bradshaw, U. Becker, H.G. Kerkhoff, B. Langer, D. Szostak and R. Wehlitz, Phys. Rev. Lett. $\underline{57}$ $1707(1986)$.

27 A.D.O. Bawagan, B.J. Olsson, K.H. Tan, J.M. Chen and B.X. Yang, Chem. Phys. 164283 (1992).

28 B. Sjörgen, S. Svensson, A. Naves de Brito, N. Correia, M.P. Keane and C. Enkvist, J. Chem. Phys. 966389 (1992).

29 S.J. Desjardins, A.D.O. Bawagan and K.H. Tan, Chem. Phys. Lett. 196 261 (1992).

30 S.J. Desjardins, A.D.O. Bawagan, K.H. Tan, Y. Wang and E.R. Davidson, Chem. Phys. Lett. (1994) (in press). 
31 S.J. Desjardins. A.D.O. Bawagan. K.H. Tan. Z.F. Liu, Y. Wang and K.R. Davidson. (1994) (to be published).

32 "Zur Encrgicabhängigke it con Photoclektronensatelliten". B. Langer (PhD) Thesis), AMS Press, New York, 1992.

33 T. Åluerg, Phys. Rev. 15635 (1967).

34 C.D. Lin, Phys. Rev. A $\underline{9} 171$ (1974).

35 A.D. Baker, C. Baker, C.R. Brundle and D.W. Turner, Int. J. Mass Spec. Ion Phys. 1285 (1968).

36 C.R. Brundle, M.B. Robin, H. Basch, M. Pinsky and A. Bond, J. Am. ('he'm. Soc. $\underline{92} 3863$ (1970).

37 G.R. Branton, D.C. Frost, T. Miakita, C.A. McDowell and I.A. Stenhouse, J. Chem. Phys. $\underline{52} 802(1970)$.

38 G.R. Branton, D.C. Frost, T. Mak'a a, C.A. Mc'Jowell and I.A. Stenhouse, Phil. Trans. R. Soc. Londors Ser. A 26877 (1970).

39 "Molecular Photoelectron Spectroscopy", D.W. Turner, C. Baker, A.I). Baker and C.R. Brundle, Wiley-Interscience, London, 1970.

40 C.R. Brundle and D.B. Brown, Spectrochim. Acta 27A 2491 (1971).

41 C. Fridh, L. Åsbrink and E. Lindholm, Chem. Phys. Lett. $\underline{15} 282$ (1972). 
I2 A. Berndtsson. B. Basilier. IV. Gelius, J. Hedman. M. Klasson. R. Nilsson. ( . Nordling and S. Svenson, Phys. Scrip. 12 235 (1975).

$4: 3$ M.S. Banna and D.A. Shirley, J. Elec. Spec. Relat. Phenom. 8255 (1976).

44 J. ('arlier and R. Botter, J. Elec. Spec. Relat. Phenom. 1791 (1979).

45 G. Bieri and L. Âsbrink, J. Elec. Spec. Relat. Phenom. 20149 (1980).

46 M.P. Keane, A. Naves de Brito, N. Correia, S. Svensson, L. Karlsson, B. Wannberg, U. Gelius, S. Lunell, W.R. Salareck, M. Lögdlund, D.B. Swanson and A.G. MacDiarmid, Phys. Rev. B $\underline{45} 6390$ (1992).

47 H. Berthod, Compt. Rend. $\underline{249} 1354$ (1959).

48 R. Hoffmann, J. Chem. Phys. 39 1397 (1963).

49 J.W. Moskowitz, J. Chem. Phys. $\underline{43} 60$ (1965).

50 W.E. Palke and W.N. Lipscomb, J. Am. Chem. Soc. $\underline{88} 2384$ (1966).

51 J.L. Whitten, J. Chem. Phys. 444359 (1966).

52 M.B. Robin, R.R. Hart and N.A. Kuebler, J. Chem. Phys. 441803 (1966).

53 J.L. Schulman, J.W. Moskowitz and C. Hollister, J. Chem. Phys. $\underline{46} 2759$ $(1967)$

54 U. Kaldor and I. Shavitt, J. Chem. Phys. $\underline{48} 191$ (1968). 
5.5 E. ('lementi and II. Popkie. J. ('hem. Phys. ji 1870 (1972).

56 1. Fischer-Hjalmars and P. Siegbahn. Theor. ('him. Acta 31 I (I!73).

5i "Bibliography of ab initio .Mole cular IIarefunctions". W.(i. Richards, T.E.H. Walker. L. Farnell and P.R. Scott. Clarendon Press, Oxford, 19i.t.

58 W. von Niessen, G.H.F. Diercksen, L.S. Cederbaum and W. Domcke, ('liem. Phys. $18469(1976)$.

59 R.L. Martin and E.R. Davidson, Chem. Phys. Lett. 51 237 (1977).

60 C.W. Murray and E.R. Davidson, Chem. Phys. Lett. 190 2:31 (1992).

61 L.S. Cederbaum, W. Domke, J. Schirmer, W. von Niessen, (i.H.F I Diercksen and W.P. Kraemer, J. Chem. Phys. 691591 (1978).

62 J. Baker, Chem. Phys. Lett. 101136 (1983).

63 H. Nakatsuji, J. Chem. Phys. $\underline{80}$ 3703 (1984).

64 H. Wasada and K. Hirao, Chem. Phys. 138277 (1989).

65 E. Lindholm and L. Åsbrink, J. Elec. Spec. Relat. Phenom. 18121 (1980).

66 T.A. Koopmans, Physica 1104 (1933).

67 M.A. Coplan, A.L. Migdall, J.H. Moore and J.A. 'Tossell, J. Am. ('hem. Soc. $1005008(1978)$. 
Gis A.J. Dixom, S.T. Hood. E. Weigold and G.R.J. Williams, J. Elec. Spec. Relat. Phenom. 11 267 (1978).

(69) I.E. Mc('arthy, J. Elec. Spec. Relat. Phenom. 3637 (1985).

70 A.S. Kheifets and M.Ya. Amusia, Phys. Rev. A 16 1261 (1992).

71 S. Braidwood, M. Brunger and E. Weigold, Phys. Rev. A 472927 (1993).

72 A.I.O. Bawagan, J. Physique 3175 (1993).

73 Z.F. Liu, G.M. Bancroft, L.L. Coatsworth and K.H. Tan, Chem. Phys. Lett. $2033337(1993)$.

74 K.H. Tan, G.M. Bancroft, L.L. Coatsworth and B.W. Yates, Can. J. Phys. 60 131 (1982).

75 B.P. Tonner and E.W. Plummer, Nucl. Inst. Meth. 177153 (1980).

76 "An Invitation to See the Light", The Synchrotron Radiation Center of the University of Wisconsin - Madison, 1994.

77 P.G. Wilkinson and R.S. Mulliken, J. Chem. Phys. $\underline{23} 1895$ (1955).

78 G. Bieri, F. Burger, E. Heilbronner and J.P. Maier, Helv. Chim. Acta $\underline{60}$ $2213(1977)$

79 T. Ishihara, J. Mizuno and T. Watanabe, Phys. Rev. A $\underline{22} 1552$ (1980).

80 J.A.R. Samson, Phys. Rev. Lett. 22693 (1969). 
81 F. Wuilleumier, M.Y. Adam. ... Sanduer and 1 . Schmidt, J. Physs. (Paris) Lett. 11 L373 (1980).

82 J.L. Dehmer and D. Dill. Phys. Rev. Lett. 35 213 (1975).

83 R.E. Palmer and P.J. Rous, Rev. Mod. Phys. 6.1 383 (1992).

84 J.L. Dehmer and D. Dill in "Electron-Molecule und Pholon-Molecule ('ollisions", edited by 'T. Rescigno, V. Mckioy and B. Srhneider, Plenum Press, New York, p 225-265, 1979.

85 S. Krummacher, V. Schmidt and F. Wuilleumier, J. Phys. B 133993 (1980).

86 L.C. Lee, R.W. Carlson, D.L. Judge and M. Ogawa, J. Quant. Spec. Rad. Trans. 131023 (1973).

87 J.G. Brennan, G. Cooper, J.C. Green, M.P. Payne and (..M. Redfern, J. Elec. Spec. Relat. Phenom. $\underline{43} 297$ (1987).

88 F.A. Grimm, T.A. Whitley, P.R. Keller and J.W. Taylor, (hem. Phys. 154 303 (1991).

89 M.L. Du and J.B. Delos, Phys. Rev. Lett. $\underline{58} 1731$ (1987).

90 R.L. Martir and D.A. Shirley, J. Chem. Phys. $\underline{64} 3685$ (1976).

91 M. Mishra and Y. Öhrn, Chem. Phys. Lett. 71549 (1980).

92 R.L. Martin, B.E. Mills and D.A. Shirley, J. Chem. Phys. 643690 (1976). 
9.3 K.I. Martin and D.A. Shirley, Phys. Rev. A 131475 (1976). 

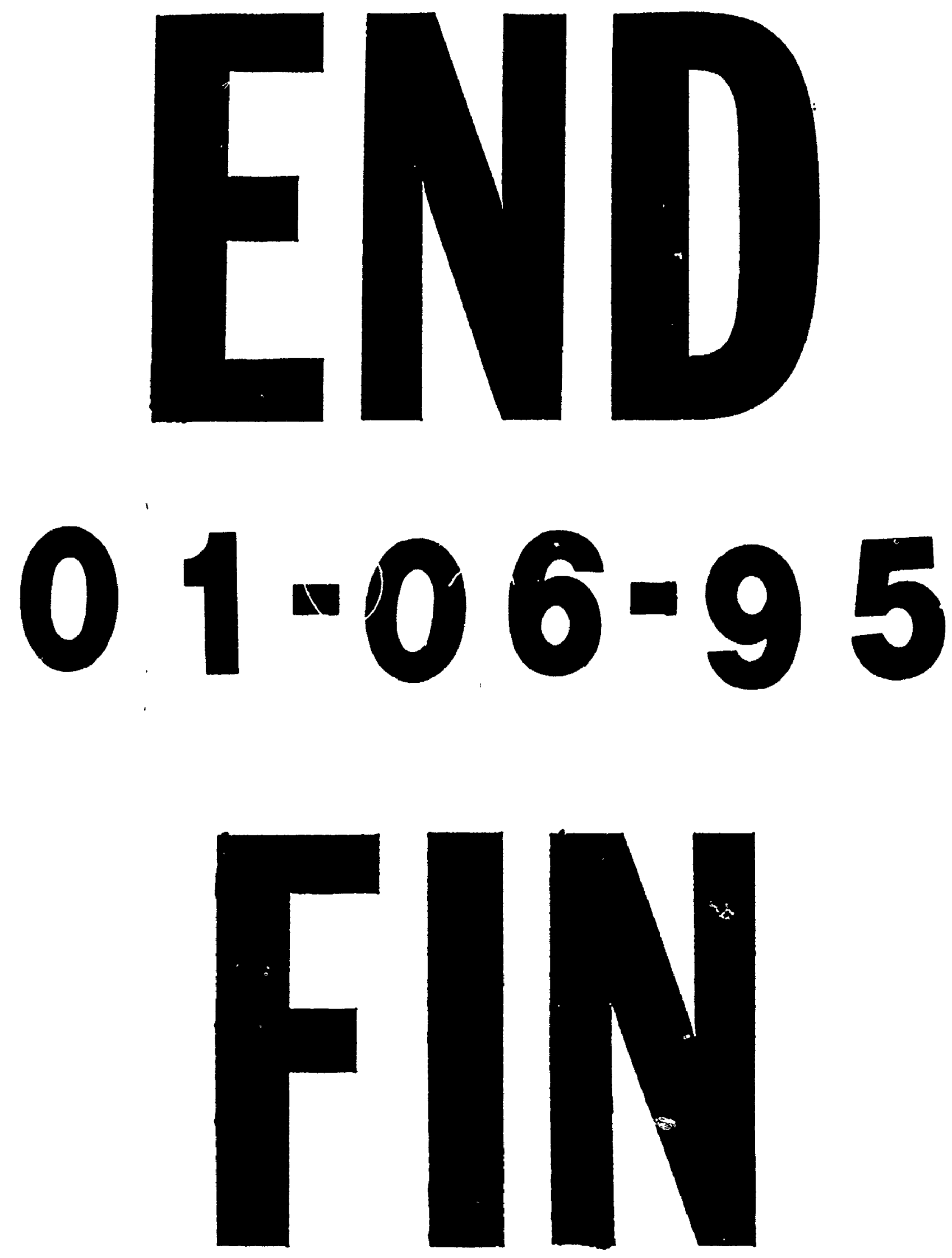